\title{
Herbivory-induced volatiles function as defenses increasing fitness of the native plant Nicotiana attenuata in nature
}

\author{
Meredith C Schuman, Kathleen Barthel ${ }^{\dagger}$, Ian T Baldwin* \\ Department of Molecular Ecology, Max Planck Institute for Chemical Ecology, Jena, \\ Germany
}

\begin{abstract}
From an herbivore's first bite, plants release herbivory-induced plant volatiles (HIPVs) which can attract enemies of herbivores. However, other animals and competing plants can intercept HIPVs for their own use, and it remains unclear whether HIPVs serve as an indirect defense by increasing fitness for the emitting plant. In a 2-year field study, HIPV-emitting $N$. attenuata plants produced twice as many buds and flowers as HIPV-silenced plants, but only when native Geocoris spp. predators reduced herbivore loads (by 50\%) on HIPV-emitters. In concert with HIPVs, plants also employ antidigestive trypsin protease inhibitors (TPIs), but TPI-producing plants were not fitter than TPI-silenced plants. TPIs weakened a specialist herbivore's behavioral evasive responses to simulated Geocoris spp. attack, indicating that TPls function against specialists by enhancing indirect defense.

DOI: 10.7554/eLife.00007.001
\end{abstract}

*For correspondence: baldwin@ ice.mpg.de

†'Present address: Federal Research Center for Cultivated Plants, Institute for Breeding Research on Horticultural And Fruit Crops, Julius Kühn Institute, Dresden, Germany

Competing interests: See page 26

Funding: See page 26

Received: 7 May 2012

Accepted: 11 July 2012

Published: 15 October 2012

Reviewing editor: Detlef Weigel, Max Planck Institute for Developmental Biology, Germany

(c) Copyright Schuman et al. This article is distributed under the terms of the Creative Commons Attribution License, which permits unrestricted use and redistribution provided that the original author and source are credited.

\section{Introduction}

Plant indirect defenses are traits that disable or remove herbivores by manipulating tri-trophic interactions to the advantage of the plant (Price et al., 1980). They attract and inform the third trophic level, predators or parasitoids, resulting in increased attacks on herbivores (Turlings and Wåckers, 2004). Indirect defenses are widespread and include domatia, extrafloral nectar, and food bodies which provide shelter and nutrition for predators and parasitoids, as well as herbivory-induced plant volatiles (HIPVs) which convey information about feeding herbivores (Heil, 2008). Field studies with the native tobacco Nicotiana attenuata, a desert annual, and with maize have shown that HIPVs can reduce herbivore loads by $24 \%$ to more than $90 \%$, by increasing both predation and parasitization of herbivores (Kessler and Baldwin, 2001; Rasmann et al., 2005; Halitschke et al., 2008; Degenhardt et al., 2009; Allmann and Baldwin, 2010) and deterring herbivore oviposition (Kessler and Baldwin, 2001).

If HIPVs really function as defenses, they should increase Darwinian fitness, defined as successful reproduction, for plants under herbivore attack (Karban and Baldwin, 1997). But because HIPVs can be perceived by many other members of the ecological community-from herbivores, pollinators, predators and parasitoids to competing or parasitic plants-it is not clear whether HIPVs increase plant fitness in nature (Dicke and Baldwin, 2010; Kessler and Heil, 2011). The field studies described above have either spanned too short a time to reveal Darwinian fitness benefits, or have not reported fitness data at all (Kessler and Baldwin, 2001; Rasmann et al., 2005; Halitschke et al., 2008; Degenhardt et al., 2009; Allmann and Baldwin, 2010). Two laboratory studies showed that parasitization of herbivores can increase plant reproduction (van Loon et al., 2000; Hoballah and Turlings, 2001), but the parasitization in these studies was not mediated by HIPVs. Hence three decades after their description, it remains unclear whether HIPVs are really indirect defenses.

Long-term field studies comparing HIPV-emitting vs -deficient plants are required in order to demonstrate a defensive function for HIPVs. Experimental additions of pure volatiles or mixes to 
eLife digest As the population of the world continues to increase beyond 7 billion, and agricultural pests continue to rapidly evolve resistance to pesticides, it is becoming ever more important to cultivate arable land in a way that is sustainable for both humans and the environment. A better understanding of the different mechanisms used by wild plants to deter herbivores will help to increase crop production without harming the environment.

Plants use both direct and indirect methods to fend off herbivores. Direct defense methods include the production of chemicals that are toxic to herbivores or give them indigestion, and the growth of sticky prickles and spines that can injure or kill the herbivore. Indirect defense methods, on the other hand, generally rely on the plant attracting organisms that are either predators or parasites of the herbivore.

Plants produce odors known as herbivory-induced plant volatiles (HIPVs) that are thought to offer indirect defense against herbivores by betraying their location to predators and parasites. However, HIPVs also influence other members of the ecological community, sometimes in ways that are detrimental to plants. Moreover, despite 30 years of research, no study has demonstrated that HIPVs increase the fitness of a plant, so it is unclear what they have evolved to do.

Now, a 2-year field study by Schuman et al. has shown plants that emit green leaf volatiles (which are a type of HIPV) produce twice as many buds and flowers-a measure of fitness-as plants that have been genetically engineered not to emit green leaf volatiles. This study was conducted with Nicotiana attenuata, which is a wild tobacco plant that is often targeted by Manduca sexta, a type of moth that is also known as the tobacco hornworm. Green leaf volatiles only increased plants' fitness when various species of Geocoris—a bug that preys on Manduca sexta-reduced the number of herbivores by a factor of two. This is the first evidence that HIPVs offer indirect defense against herbivores.

Schuman et al. also studied the effects of molecules called protease inhibitors that are thought to function as direct defenses by making it difficult for herbivores to digest plants. They found that the ability to produce protease inhibitors did not increase the fitness of plants under herbivore attack; however, tobacco hornworms that had been fed plants containing protease inhibitors were found to be more sluggish in response to attack, which suggests that protease inhibitors can enhance the indirect defenses of plants. The results suggest that employing both direct and indirect defenses-such as a combination of biological pesticides and genetic engineering to produce both HIPVs and protease inhibitors-is the best approach for defending agricultural plants against pests. DOI: 10.7554/eLife.00007.002

plants growing in nature has worked well to test short-term effects of specific compounds (Kessler and Baldwin, 2001; Allmann and Baldwin, 2010), but only endogenously produced HIPV emissions can ensure specific, lasting and consistent differences under field conditions. Most evidence for the utility of HIPVs comes from studies in which predators and parasitoids learn to associate HIPVs with prey; naïve predators and parasitoids are just as likely to respond to HIPVs as not to respond (Allison and Hare, 2009). Thus the inducibility of HIPV emission, which ensures association with herbivore feeding, is likely essential for HIPV function, but it is difficult to engineer (Kos et al., 2009). Engineered constitutive HIPV emissions have been used, either on predators and parasitoids trained to associate target volatiles with prey in short-term laboratory experiments (Kappers et al., 2005; Schnee et al., 2006), or in set-ups in which target volatiles are always associated with prey (Rasmann et al., 2005; Degenhardt et al., 2009). When plants are engineered constitutively to emit HIPVs, they no longer provide accurate information about the location of feeding herbivores, and predators will not associate these signals with prey in nature. Genetically silencing the biosynthesis of HIPVs, however, permits naturally inducible wild-type (WT) plants to serve as HIPV emitters, for comparison with transformed lines lacking specific volatile components (Halitschke et al., 2008; Skibbe et al., 2008). Furthermore, field experiments that manipulate the production of HIPVs which not only attract the third trophic level, but also influence the second trophic level (e.g., as feeding stimulants and host location cues), require additional experimental manipulations to preserve the plant-herbivore part of the tritrophic interaction. 
When HIPVs do attract the third trophic level, how can herbivores adapt? Many herbivores can outgrow their vulnerability to predators and parasitoids, but plant direct defenses can slow herbivore growth and prolong vulnerability as postulated by the slow growth-high mortality hypothesis (Benrey and Denno, 1997; Williams, 1999; Kessler and Baldwin, 2001, 2004; Kaplan and Thaler, 2011). The solanaceous specialists Manduca sexta and M. quinquemaculata (Lepidoptera, Sphingidae) are resistant to the potent alkaloid toxin nicotine (Wink and Theile, 2002), but sensitive to the nutritional value of plant tissue (Zavala and Baldwin, 2004). Non-toxic protease inhibitor (PI) proteins, which inhibit protein digestion and thus decrease the availability of organic nitrogen in the form of amino acids (Zavala et al., 2008), are widespread in flowering plants (Hartl et al., 2011), and trypsin protease inhibitors (TPIs) slow the growth of M. sexta on N. attenuata (Zavala et al., 2008). However, herbivores can overcome Pls by producing insensitive or desensitized proteases, inactivating or degrading Pls, eating more plant tissue, and eating more nutritious young tissue (Winterer and Bergelson, 2001; Steppuhn and Baldwin, 2007; Zavala et al., 2008). In the latter two cases, Pls could reduce plant fitness. Although TPI-producing N. attenuata plants produce more seeds than TPI-deficient plants when attacked by M. sexta under controlled glasshouse conditions (Zavala and Baldwin, 2004), whether TPIs function as a direct defense in nature is unknown.

We tested the hypotheses that HIPVs and TPIs defend plants in nature by increasing herbivore predation and thereby plant Darwinian fitness. To do so, we monitored the performance, predation and mortality of Manduca spp. ( $M$. sexta and M. quinquemaculata) on wild-type $N$. attenuata plants and RNAi transformed lines silenced for the production either of a specific group of HIPVs, or of TPIs, and compared the resulting plant reproductive output in terms of bud and flower production (we are not permitted to allow transgenic plants to disperse ripe seed). Because N. attenuata is an annual, opportunistic out-crosser, seeds are produced within one growing season, mostly from fertilization via self-pollen (Sime and Baldwin, 2003), and we can relate bud and flower production to lifetime seed production, which is commonly accepted as a measure of Darwinian fitness (Baldwin, 1998; van Loon et al., 2000; Hoballah and Turlings, 2001).We hypothesized that HIPVs would increase plant reproduction by increasing predation of herbivores, and that TPIs alone would not increase reproduction under herbivore attack, but would either increase predation or increase herbivores' susceptibility to predators. We then assembled a toolbox of wild-type and transgenic lines chosen to test these hypotheses.

We chose a genotype of $N$. attenuata native to the Great Basin Desert of southwestern Utah. In many years, Manduca spp. larvae cause the most defoliation of $N$. attenuata plants in this area (Kessler and Baldwin, 2001) and thus the N. attenuata 'UT' genotype is likely adapted to defend against Manduca spp. Eggs and young larvae of Manduca spp. are predated by Geocoris spp. (bigeyed bugs) which occur naturally in the Utah habitat and are attracted to components of N. attenuata's HIPV bouquet (Kessler and Baldwin, 2001; Halitschke et al., 2008; Skibbe et al., 2008). Specifically, Utah Geocoris spp. predators are attracted to the sesquiterpene (E)- $\alpha$-bergamotene as well as green leaf volatiles (fatty acid-derived $C_{6}$ aldehydes, alcohols and esters) (Kessler and Baldwin, 2001; Halitschke et al., 2008; Schuman et al., 2009). Green leaf volatiles, or GLVs, can be silenced via a single upstream 13-lipoxygenase, NaLOX2, which specifically supplies lipid hydroperoxides for their production (Allmann et al., 2010). Although GLVs are released upon mechanical damage, the oral secretions (OS) of $M$. sexta convert 3-(Z)-GLVs to the 2-(E)-structures, resulting in greater Geocoris spp. predation than the damage-induced (Z):(E) ratio (Allmann and Baldwin, 2010). GLVs are released immediately upon damage (Allmann and Baldwin, 2010) and may therefore be a 'first line of defense'.

Like GLVs, many other HIPVs are also released after mechanical damage, but change in amount or ratio upon herbivory, and thus GLVs mirror the functional complexity of the total HIPV blend. Furthermore, GLVs prime or directly regulate responses in neighboring plants (Kessler et al., 2006; Paschold et al., 2006), attract herbivores as well as predators (Halitschke et al., 2008), and are important cues for pollinating and ovipositing moths (Kessler and Baldwin, 2001, 2006; De Moraes et al., 2001; Fraser et al., 2003), thus performing several roles which may harm or benefit plant fitness in addition to their role in attracting predators. Perhaps most significantly, GLVs also stimulate Manduca spp. feeding, and silencing plant GLV production results in reduced herbivore damage (Halitschke et al., 2004; Meldau et al., 2009). All these qualities made the manipulation of GLV emissions an ideal means to test rigorously the fitness consequences of HIPV emissions and to evaluate whether these emissions can truly be considered defensive. 


\section{Results}

\section{GLV and TPI production are reduced or eliminated in transformed lines}

We chose a line of irPI plants with no detectable TPI activity (Steppuhn and Baldwin, 2007), and a line of irLOX2 plants with GLV emissions $<20 \%$ of WT (Allmann et al., 2010); non-target defense metabolites are not affected in either line (Steppuhn and Baldwin, 2007; Allmann and Baldwin, 2010), including emission of (E)- $\alpha$-bergamotene measured in a glasshouse characterization of all lines prior to field release (see 'Non-target metabolites are not affected in irLOX2, hemi-irLOX2 or irPI plants'). Because of the importance of GLVs for the plant-herbivore interaction, we used both homozygous (Allmann et al., 2010) and hemizygous irLOX2 plants to provide different levels of GLV silencing. Hemizygous (hemi-) irLOX2 plants were created by crossing homozygous irLOX2 and irPI plants, but the irPI construct was not active in this cross (Figure 1 see 'Discussion').

The irPl plants (Steppuhn and Baldwin, 2007) had no detectable TPI activity in the glasshouse or throughout the field experiment in 2011, and PI transcripts accumulated to only $0.3 \%$ of WT levels in irPI (transcripts, $N=5, p<0.001$ in Scheffe post hoc tests following two-way ANOVAs on log $_{2}$-transformed data with factors $\mathrm{W}+\mathrm{OS}$ treatment and genotype: treatment $\mathrm{F}_{1,29}=75.909, \mathrm{p}<0.001$; genotype $\left.F_{3,29}=174.077, p<0.001\right)$; in contrast, TPI activity and $P$ I transcripts were similar to WT plants in irLOX2 and hemi-irLOX2 (transcripts, $N=5, p>0.2$ in Scheffe post hoc tests following two-way ANOVAs on $\log _{2}$-transformed data with factors $\mathrm{W}+\mathrm{OS}$ treatment and genotype; activity, $\mathrm{N}=10-17, \mathrm{p}>0.05$ in oneway ANOVAs with factor genotype) (Figure 1).

We assessed GLV production by hexane extraction of GLVs from frozen leaf tissue, and GLV emission by GC analyses of leaf headspaces. GLVs in hemi-irLOX2 plants were reduced to levels similar to those in irLOX2 plants, but hemi-irLOX2 plants still produced detectable amounts of (Z)-3-hexenol (Figures 2 and 3). The dominant GLV in hexane tissue extracts was (E)-hex-2-enal, and (Z)-hex-3-en1 -ol was additionally quantifiable as a minor component. Only (E)-hex-2-enal was quantifiable in extracts from field-grown plants on May 28, 2011, and was below quantifiable levels in irLOX2 and hemi-irLOX2 plants, but detectable in pooled samples from hemi-irLOX2 (Figures 2 and 3). Extracts from later in the season also contained quantifiable amounts of (Z)-hex-3-en-1-ol and hemi-irLOX2 extracts contained up to $50 \%$ as much of this alcohol as WT and irPl extracts $(N=10, p<0.05$ in Scheffe post hoc tests following one-way ANOVAs with factor genotype: June 14, 2011, $F_{2,26}=9.556$, $p=0.001$; June 22, 2011, $F_{2,26}=12.196, p<0.001 ; p>0.6$ for irPI vs WT in a t-test for May 28 and in Scheffe post hoc tests for June 14 and 22) (Figure 3). Headspace measurements from field- and glasshouse-grown plants detected a similar $80-100 \%$ reduction in GLV emissions from irLOX2 and hemi-irLOX2 plants compared to WT and irPI (field, N=3, $\mathrm{p}=0.024$ for hemi-irLOX2 v irPl, $\mathrm{p}>0.05$ for hemi-irLOX2 vs WT and irLOX2 vs irPI and WT, but $p=0.939$ for irPI vs WT in Scheffe post hoc tests following one-way ANOVA: $F_{3,8}=7.346, p=0.011$; glasshouse, $N=4$ : irLOX2 and hemi-irLOX2 below limit of detection, $p=0.834$ for t-test irPI vs WT) (Figure 3), and transcript accumulation of LOX2 was $2 \%$ of WT levels in irLOX2 and hemi-irLOX2 $(N=5, p<0.001$ in Scheffe post hoc tests following twoway ANOVAs on $\log _{2}$-transformed data with factors $\mathrm{W}+\mathrm{OS}$ treatment and genotype: treatment $F_{1,32}=0.021, p=0.887$; genotype $\left.F_{3,32}=635.477, p<0.001\right)$ but unaffected in ir $P I$ ( $p>0.9$ in Scheffe post hoc test vs WT) (Figure 3 ).

\section{Non-target metabolites are not affected in transformed lines}

For the 'UT' genotype of $N$. attenuata used in our experiments, the induction of all HIPVs except GLVs is mediated by jasmonate signaling (Halitschke and Baldwin, 2003; Kessler et alo, 2004). The irPI line A-04-186-1 (Steppuhn and Baldwin, 2007) and irLOX2 line A-04-52-2 (Allmann et al., 2010) have been characterized previously, and neither is affected in jasmonate signaling. Particularly, the emission of (E)- $\alpha$-bergamotene, the best-characterized HIPV in N. attenuata apart from GLVs (Halitschke et al., 2000; Kessler et al., 2004; Halitschke et al., 2008; Skibbe et al., 2008), does not differ significantly among the lines used ( $N=4$ measured 24-32 hr after $\mathrm{W}+\mathrm{OS}$ treatment as according to Halitschke et al. (2000) and normalized as a percentage of the

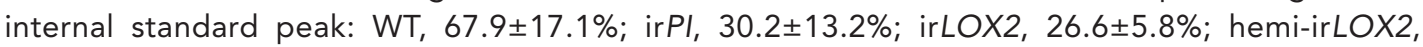
$42.7 \pm 23.3 \%$; ANOVA: $F_{3,12}=1.338, p=0.308$ ). The transformation process itself does not affect plant fitness or competitive ability (Schwachtje et al., 2008), TPI production or volatile emission (Figures 1-3). 


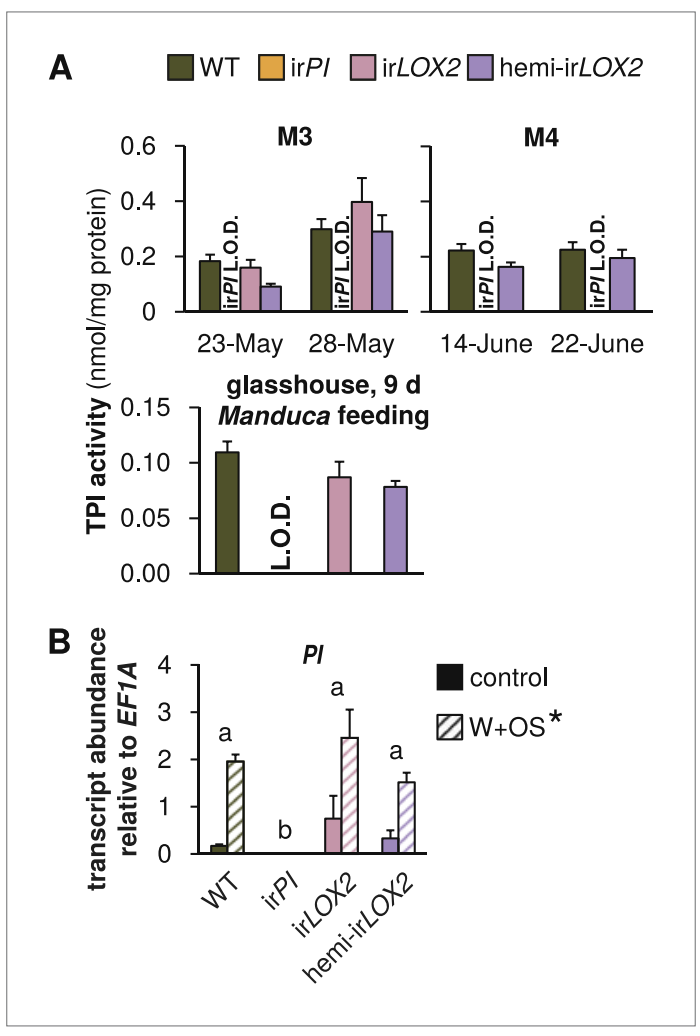

Figure 1. Trypsin protease inhibitor (TPI) activity and transcripts in transformed lines; graphs show means+SEM. (A) TPI activity measured in systemic leaves of field-grown (top two panels, 2011, N=11-14 for panel 1 and $\mathrm{N}=21$ for panel 2) or glasshouse-grown plants (bottom panel, $\mathrm{N}=10$ ) attacked by Manduca spp. larvae. Only WT, irPI and hemi-irLOX2 plants were used in M4. For a timeline of Manduca spp. infestations M1-M4 see Figure 4A. For raw data, see F2A SchumanBarthelBaldwin2012TPlactivity.xlsx (Dryad: Schuman et al., 2012). (B) Transcripts of $P I$ in unelicited leaf tissue (control), and at the point of maximum accumulation in $\mathrm{W}+\mathrm{OS}$-elicited leaf tissue in glasshouse-grown plants $(\mathrm{N}=5)$. For raw data, see F2B_ SchumanBarthelBaldwin2012PItranscripts.xIsx (Dryad: Schuman et al., 2012). ${ }^{*} W+O S$ treatment had a significant effect on $\mathrm{PI}(p<0.001)$ transcript accumulation. ${ }^{a, b}$ Different letters indicate significant differences between genotypes $(p<0.001)$ in Scheffe post hoc tests following a two-way ANOVA on $\log _{2}$-transformed data with factors treatment and genotype (genotype $F_{3,29}=174.077, p<0.001$; treatment $\left.F_{1,29}=75.909, p<0.001\right)$. L.O.D.: below limit of detection for measurement. DOI: 10.7554/eLife.00007.003

into the field on average 3 days earlier than irLOX2 and hemi-irLOX2 plants, so that all plants were planted at a similar size, which is important for even establishment. We therefore re-infested WT and irPI plants earlier after M2, to allow irLOX2 and hemi-irLOX2 plants to catch up in their growth to WT and irPI before re-infestation, so as not to bias further assays. However, we left $M$. sexta larvae on irLOX2 and hemi-irLOX2 as long as on WT and irPl, and we made several control measurements to ensure that differences in Geocoris spp. predation were not due to our staggering of infestation: we counted Geocoris spp. populations around all genotypes over this period (Table 2) and saw that they

\section{Geocoris spp. consistently prefer to predate from GLV-perfumed or -emitting plants}

We monitored the predation of Manduca spp. larvae and eggs daily, and counted Geocoris spp. individuals around plants every 2-3 days (Geocoris spp. counts, Figure 4). In 2010, we planted into a first-year plot. Although plants were infested with laboratory strain $M$. sexta larvae $(N=51)$ and baited with $M$. sexta eggs $(N=50)$ over a 2-week period in 2010 (Figure 4), no Geocoris spp. individuals were observed on this plot through May. There were also no Geocoris spp. observed through May on a nearby, older plot: Geocoris spp. first arrived and began to predate Manduca spp. eggs on the older plot on June 9. In 2011, we planted into the older plot, where we observed Geocoris spp. in May prior to the first infestation (M2, Figure 4).

During infestation M2 (Figure 4), we allowed Geocoris spp. to associate all four plant genotypes with the presence of prey: we infested half of all plants with equal numbers of first-instar $M$. sexta larvae from the laboratory strain and, because Geocoris spp. predate more from GLV-emitting orperfumed plants (Kessler and Baldwin, 2001; Halitschke et al., 2008; Allmann and Baldwin, 2010), we supplemented GLV emission from irLOX2 and hemi-irLOX2 plants by placing cotton swabs with lanolin paste containing GLVs representative of the M. sexta-fed $N$. attenuata headspace (Table 1 Allmann and Baldwin, 2010) adjacent to $M$. sexta-infested leaves. Swabs containing lanolin with solvent were placed next to ir Pl and WT as a control. M. sexta larvae were predated at a rate of $12-37 \%$ over two 2 - to 3 -day trials. Geocoris spp. tended to predate more larvae from GLV-supplemented plants (Fisher's exact tests, $35-37 \%$ vs $22-27 \%$ May $5-6, \mathrm{~N}=59-60$ larvae, $\mathrm{p}=0.066 ; 17-21 \%$ vs $12 \%$ May $13-15$, $\mathrm{N}=92-100$ larvae, $\mathrm{p}=0.069$; combined trials, Bonferroni-corrected $\mathrm{p}=0.0063$ ) (Figure 5).

We removed the cotton swabs and the remaining larvae. We then monitored predation of newly-infested larvae and eggs without GLV supplementation during infestation M3 (Figure 4). We staggered infestation to accommodate differences in plant growth: WT and irPl seedlings were initially larger and therefore were planted 


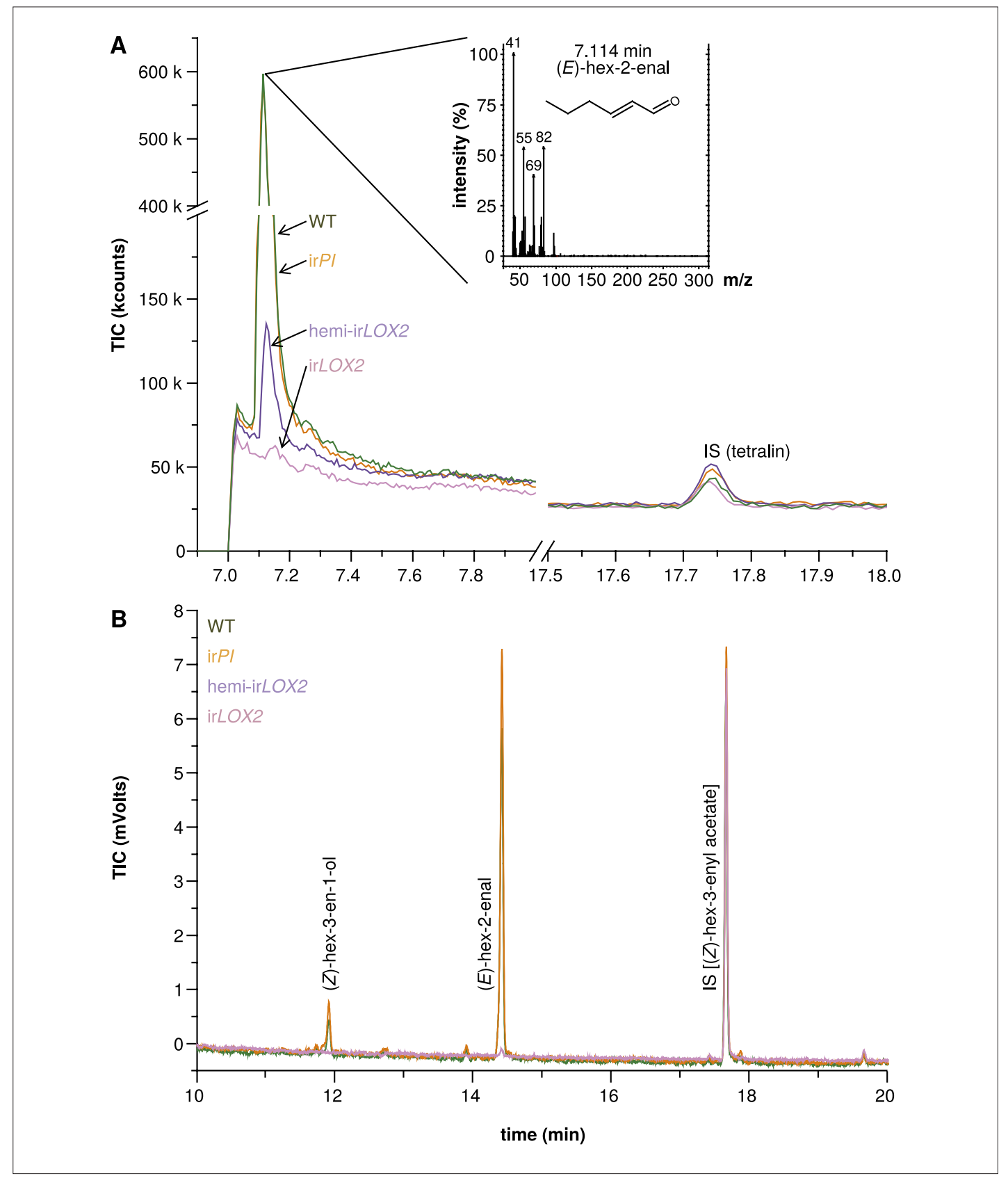

Figure 2. Hexane extracts of leaves from field-grown plants. (A) Hexane extracts from pooled leaf samples of field-grown plants for a qualitative assessment of green leaf volatile (GLV) pools, analyzed by GC-MS with a split ratio of 1/100 onto a nonpolar column; only (E)-hex-2-enal was identified due to poor resolution of (E)-hex-2-enal and (Z)-hex-3-en-1-ol on the nonpolar column; no ester peaks were detected. For raw data, see F3A_SchumanBarthelBaldwin2012chromatograms.xlsx (Dryad: Schuman et al., 2012). (B) Example chromatograms from hexane extracts of individual leaf samples from field-grown plants, analyzed by GC-FID on a wax column. The dominant compound was (E)-hex-2-enal; (Z)-hex-3-en-1-ol was also present in quantifiable amounts. (Z)-3-hexenyl acetate was chosen as an internal standard because no esters were detectable in the preliminary qualitative GC-MS analysis ( $1 \mathrm{~A})$, and because its chemical similarity to (E)-hex-2-enal and (Z)-hex-3-en-1-ol made it a good choice of internal standard for normalization and calculation of yield from extracts. For raw data, see F3B_SchumanBarthelBaldwin2012chromatograms.xlsx (Dryad: Schuman et al., 2012). IS: internal standard. DOI: 10.7554/eLife.00007.004 
A $\square(E)$-hex-2-enal $\square(Z)$-hex-3-en-1-ol

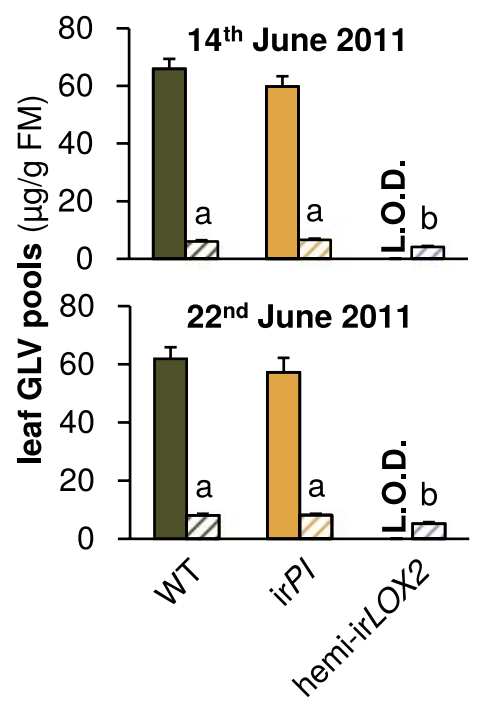

B Total GLVs

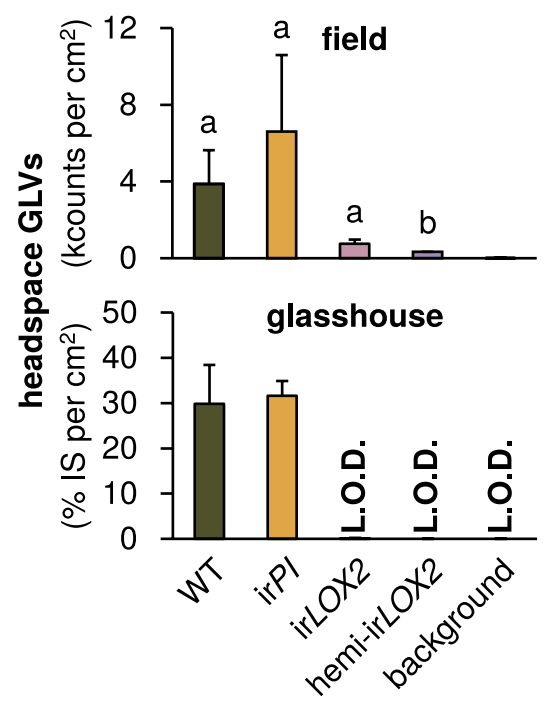

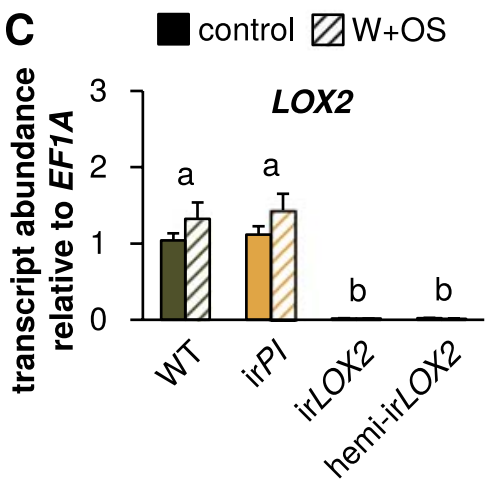

Figure 3. GLV production and emission in transformed lines; graphs show means+SEM. (A) GLVs extracted with hexane from leaf tissue of fieldgrown WT, irPl, and hemi-irLOX2 plants grouped in triplets for infestation M4 in 2011(Figure 4A). Leaves were harvested from every plant at the beginning (June 14) and in the middle of M4 (June 22) and leaves from plants in 10 randomly chosen triplets were analyzed. Only (E)-hex-2enal and (Z)-hex-3-en-1-ol were quantifiable in leaf extracts. Different letters ( $a$ and b) indicate significant differences $(p \leq 0.05)$ in Scheffe post hoc tests following one-way ANOVAs for (Z)-hex-3-en-1-ol (top panel, $F_{2,26}=9.556, p=0.001$; bottom panel, $F_{2,26}=12.196$, $p<0.001$ ). For raw data, see F4A_ SchumanBarthelBaldwin2012GLVpools.xlsx (Dryad: Schuman et al., 2012). (B) GLVs measured in headspace samples of leaves from field-grown (top panel, $\mathrm{N}=3$ ) or glasshouse-grown plants (bottom panel, $\mathrm{N}=4$ ). For field-grown plants, leaves were harvested and measured on May 21 (just before $\mathrm{M} 3$ ). Intact leaves were kept fresh by placing petioles in water. Immediately before each measurement, one leaf was treated with wounding and $M$. sexta oral secretions (W+OS); a 1-cm² disc was stamped out and placed in a 4-mL GC vial. After $15 \mathrm{~min}$ the headspace in the vial was measured with a Z-Nose 4200 and total alcohols and aldehydes were quantified. Different letters $(a$ and $b)$ indicate significant differences $(p<0.05)$ in Scheffe post hoc tests following one-way ANOVA $\left(F_{3,8}=7.346, p=0.011\right)$. For glasshouse-grown plants, leaves were left on plants, treated with $\mathrm{W}+\mathrm{OS}$, and enclosed in padded, $50 \mathrm{~mL}$ food-quality plastic containers for $3 \mathrm{hr}$ while the headspace was pulled over a Poropak $\mathrm{Q}$ filter. Filter eluents were measured by GC-MS. Three-hour headspace samples contained (Z)-hex-3-en-1-ol, (E)-hex-2-en-1-ol (forms from (E)-hex-2-enal on filters over trapping periods longer than 20 min), (Z)-hex-3-enyl acetate, (Z)-hex-3-enyl butanoate, (Z)-hex-3-enyl isobutyrate, and (Z)-hex-3-enyl propanoate, all of which showed the pattern shown for the total amount. For raw data, see F4B_SchumanBarthelBaldwin2012GLVheadspace.xlsx (Dryad: Schuman et al., 2012). (C) Transcripts of LOX2 in unelicited leaf tissue (control), and at the point of maximum accumulation in $\mathrm{W}+\mathrm{OS}$-elicited leaf tissue in glasshouse-grown plants ( $\mathrm{N}=5)$. For raw data, see F4C_SchumanBarthelBaldwin2012LOX2transcripts.xIsx (Dryad: Schuman et al., 2012). a, b Different letters indicate significant differences

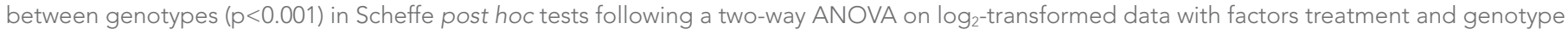
(genotype $F_{3,32}=635.477, p<0.001$, treatment $F_{1,32}=0.021, p=0.887$ ). L.O.D.: below limit of detection for measurement. DOI: 10.7554/eLife.00007.005

were not different ( $p>0.05$ in Fisher's exact tests), indicating that Geocoris spp. continued to explore irLOX2 and hemi-irLOX2 plants but not to predate from them over a week of infestation followed by 5 days of $M$. sexta egg predation assays (during which $M$. sexta eggs were simultaneously applied to all genotypes); and we followed predation of $M$. sexta larvae from all four genotypes in parallel over 1 week, during which irLOX2 and hemi-irLOX2 were infested with more larvae than WT and irPI due to sustained higher predation rates on WT and irPl; but predation remained higher on WT and irPl.

Predation of both larvae and eggs without GLV supplementation was two to four times as great on GLV-emitting WT and irPI plants: 43\%/60\% (WT/irPI) for larvae and 34\%/39\% for eggs, vs 17\%/33\% (irLOX2/hemi-irLOX2) for larvae and 9\%/20\% for eggs (Fisher's exact tests: $N=30$ larvae, $p=0.047$ for irLOX2 vs WT, $p=0.069$ for hemi-irLOX2 vs irPl; $N=88$ eggs, $p<0.001$ for irLOX2 vs WT, $p=0.013$ for hemi-irLOX2 vs irPI) (Figure 5). Predation was associated with a steady Geocoris spp. population of 16-23 individuals per day within a $5 \mathrm{~cm}$ radius around plants (Table 2). However, there was no difference among plant genotypes in the number of Geocoris spp. individuals ( $p>0.05$ in Fisher's exact tests), indicating that Geocoris spp. regularly survey all plants and use GLVs as a short-distance cue to determine which plants harbor prey. Figure 5 shows larval predation rates at the beginning of the 
A 2010: no Geocoris spp. predation

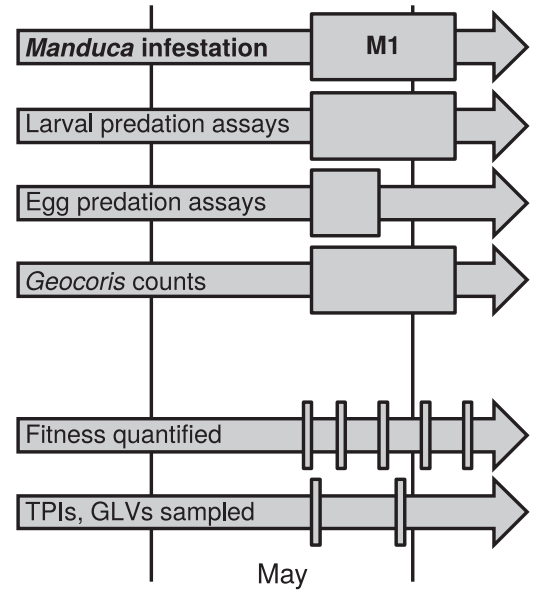

2011: Geocoris spp. predation

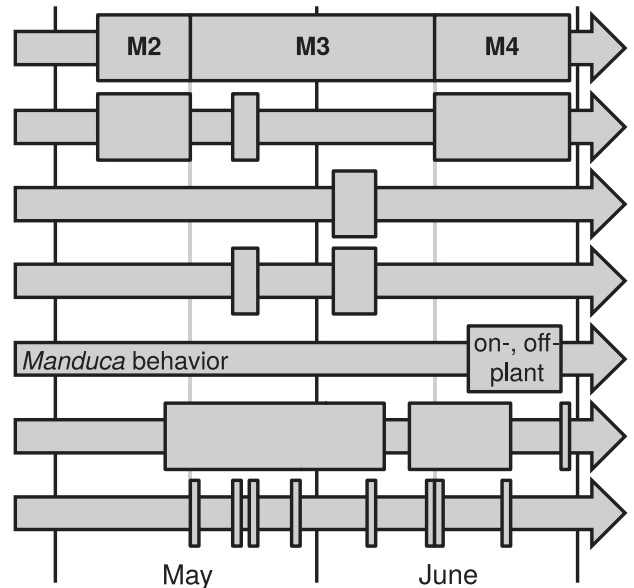

\section{B: 2010}

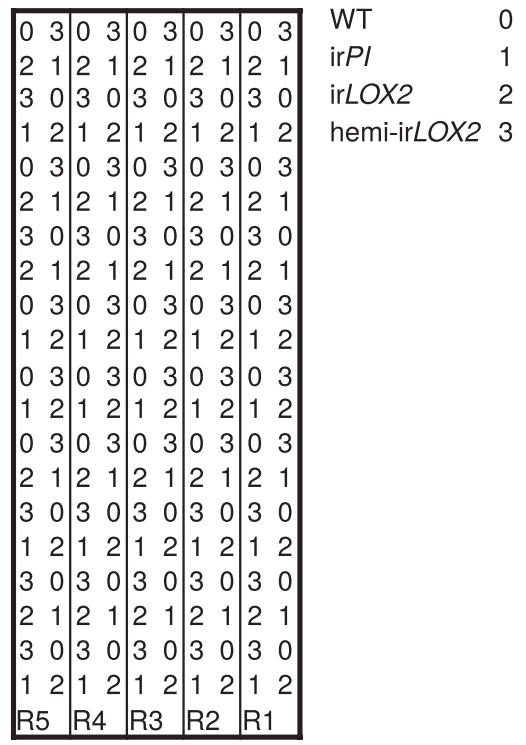

\section{C: 2011}

\begin{tabular}{llllllllllll}
\hline R1 & R2 & R3 & R4 & R5 & R6 & R7 & R8 & R9 R10 R11 R12 R13 R14 R15 R16 R17R18 R19 R20
\end{tabular}

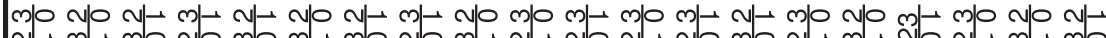

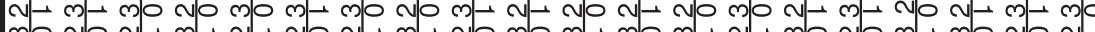

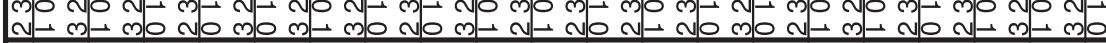

Figure 4. Experimental timeline and layout. (A) Timeline of field experiments in 2010 and 2011. Different assays and measurements are represented by individual arrows, and rectangles span the time frame of each assay or measurement; narrow rectangles represent single days. Four experimental Manduca infestations (M1-M4) structure the overall experimental design: M1-M3, with laboratory Manduca, and M4, with wild Manduca larvae. (B and C) Layouts of field plots in (B) 2010 and (C) 2011. Thick lines denote the borders of the experiment, thin lines denote irrigation lines (vertical borders of plot were also irrigation lines in [B] 2010), and R\# denotes row number (used for identifying replicates during the experiment). The genotype key in (B) applies to both (B) and (C).

DOI: 10.7554/eLife.00007.006 
Table 1. GLV mix used to externally supplement plant GLV emission in M2 (see Figure 4) (Allmann and Baldwin, 2010)

\begin{tabular}{ll} 
Component & Nanogram/20 $\boldsymbol{\text { LL lanolin }}$ \\
\hline (Z)-hex-3-enal & 3530 \\
\hline (E)-hex-2-enal & 2690 \\
\hline (Z)-hex-3-en-1-ol & 1780 \\
\hline$($ E)-hex-2-en-1-ol & 2440 \\
\hline$(Z)$-hex-3-enyl acetate & 46.6 \\
\hline (E)-hex-2-enyl acetate & 35.5 \\
\hline (Z)-hex-3-enyl propanoate & 9.00 \\
\hline (E)-hex-2-enyl propanoate & 8.08 \\
\hline (Z)-hex-3-enyl butanoate & 97.0 \\
\hline (E)-hex-2-enyl butanoate & 35.6
\end{tabular}

Pure GLVs were diluted in $1 \mathrm{~mL}$ of hexane and mixed into $14 \mathrm{~mL}$ of lanolin to yield the amount shown per $20 \mu \mathrm{L}$, representing the emission per $\mathrm{g}$ leaf material within the first 20 minutes of $\mathrm{W}+\mathrm{OS}$ elicitation. Lanolin containing an equivalent amount of hexane was used as a control.

DOI: 10.7554/eLife.00007.007

assay, when the M. sexta load was comparable across plant genotypes. Over the following week, Geocoris spp. predated a total of $80 \%$ of these larvae from WT and irPI vs $47 \%$ from irLOX2 (Fisher's exact test, $p=0.015$ vs WT) and $67 \%$ from hemi-irLOX2 ( $p=0.382$ vs irPI).

In summary, Geocoris spp. had the same opportunity to locate $M$. sexta larvae and eggs on all genotypes, but consistently preferred to predate from GLV-supplemented or -emitting plants.

\section{Manduca spp. damage reduces plant growth and reproduction}

We took the different number of 'days in field' for each plant into account in our comparison of growth and reproduction among genotypes and therefore the staggered planting did not affect this comparison (Figure 6, statistics Table 3). The irLOX2 and hemi-irLOX2 plants suffered, in total, a similar amount of M. sexta damage to WT plants in trials M2 and M3 (Figure 4), and only irPl plants suffered significantly less M. sexta damage (Figure 7 ).

Reduced predation of M. sexta from irLOX2 and hemi-irLOX2 in trials M2 and M3 in 2011 (Figure 4) correlated with the reduced growth and reproduction of both genotypes, by $30-50 \%$ for irLOX2 and 20-30\% for hemi-irLOX2 vs WT, although this reduction was also apparent in plants not infested with M. sexta. (Figures 4 and 6, statistics Table 3). In 2010 however, in the absence of predation, there was no difference in stem growth, branching, or bud and flower production among genotypes irrespective of $M$. sexta infestation (Figure 6, statistics Table 3). Although $M$. sexta feeding significantly affected growth and reproduction of plants overall, the effect was not significant for irLOX2 or irPI plants in either year (Figure 6, statistics Table 3), possibly due to reduced feeding damage resulting from a lack of TPI-induced compensatory feeding in irPI (Steppuhn and Baldwin, 2007) (Mann-Whitney U-test between irPI and WT on May 28, U=54, $p=0.046$, Figure 7). Although GLVs are feeding stimulants (Halitschke et al., 2004), we could not measure reduced $M$. sexta feeding damage in hemi-irLOX2 or irLOX2 (Figure 7). Yet hemi-irLOX2 plants, despite strongly reduced GLVs, still suffered reduced growth and reproduction from Manduca spp. feeding: M. sexta feeding reduced flower production rates by about $50 \%$ in WT and by about $30 \%$ in hemi-irLOX2 plants in 2010, although the overall reduction was only significant in WT; and reduced bud production significantly for both WT and hemi-irLOX2 by $25-30 \%$ in 2011 (Figure 6, statistics Table 3).

\section{Damage from naturally occurring herbivores other than Manduca spp. cannot explain differences in plant fitness}

We monitored herbivore attack to determine whether GLV-silenced plants suffered different amounts of damage from naturally occurring herbivores, which could also cause differences in their growth and reproduction. All genotypes were attacked by mirid (Tupiocoris notatus) and noctuid herbivores 


\section{A: Predated larva and egg}

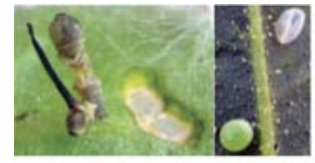

B: GLVs supplemented

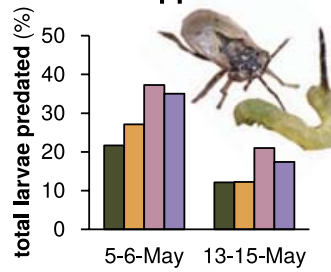

C: no supplementation

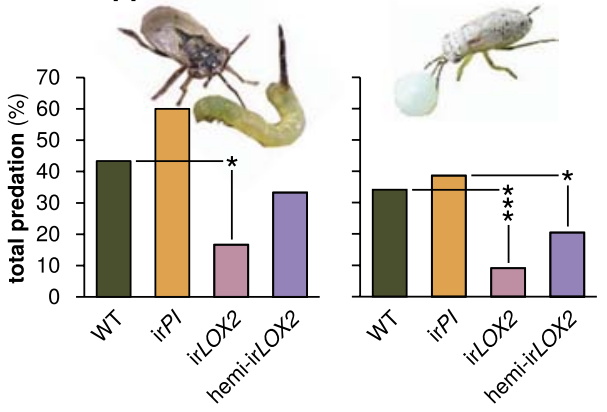

Figure 5. Predation of M. sexta larvae and eggs by Geocoris spp. (A) Examples of predated M. sexta larva (left panel) and egg (right panel). Left, the carcass of a predated first-instar M. sexta larva and typical feeding damage from early-instar Manduca spp. larvae. Right, an intact (lower left) and a predated (upper right) Manduca spp. egg. In this case, the predated egg collapsed during predation. (B) Total predation of M. sexta larvae per trial over two trials during infestation M2. GLVs were supplemented externally by placing cotton swabs next to Manduca-infested leaves (1 per plant). Cotton swabs next to irLOX2 and hemi-irLOX2 plants received $20 \mu \mathrm{L}$ of a GLV mixture in lanolin paste (Table 1); those next to WT and irPI plants received lanolin with hexane as a control because hexane was used to dissolve GLVs before mixing with lanolin. $\mathrm{N}=59-60$ larvae on May 5-6 and 92-100 larvae on May 13-15. Geocoris spp. tended to predate more larvae from GLV-supplemented plants (Fisher's exact tests, 35-37\% vs 22-27\% May 5-6, $p=0.066 ; 17-21 \%$ vs $12 \%$ May $13-15, p=0.069$; combined trials, Bonferroni-corrected $\mathrm{p}=0.0063$ ). (C) Total percentage of $\mathrm{M}$. sexta larvae (left panel, $\mathrm{N}=30$ larvae) and eggs (right panel, $\mathrm{N}=88$ eggs) predated in two separate trials during infestation M3 in 2011 (Figure 4). There was no predation of larvae or eggs by Geocoris spp. in 2010. Raw data for (B) and (C) is in F5BC_SchumanBarthelBaldwin2012predation. xlsx (Dryad: Schuman et al., 2012). Pictures are of a G. pallens adult predating a first-instar Manduca spp. larva (left) and a fifth-instar G. pallens nymph predating a Manduca spp. egg (right picture, S. Allmann). * $p<0.05$, ${ }^{* * *} \mathrm{p}<0.001$ in Fisher's exact tests against WT (irLOX2) or irPI (hemi-irLOX2, which also contains the irPI construct). DOI: 10.7554/eLife.00007.008 which caused similar amounts of damage across genotypes and years (ca. $15 \%$ and $3 \%$ of total canopy area, respectively) although irLOX2 plants suffered $60 \%$ less mirid and noctuid damage by the end of $M 3$ in 2011 ( $N=24-28$; Bonferroni-corrected Kruskal-Wallis test, noctuids May 5, $p=0.027$, all pairwise tests Bonferronicorrected $p>0.05$; one-way ANOVAs with factor genotype on arcsine-transformed data: mirids May 27, $F_{3,103}=5.291, p=0.002, p<0.05$ for irLOX2 vs hemi-irLOX2 and irPI in Scheffe post hoc tests, noctuids May 27, $F_{3,103}=3.503$, $p=0.018$, all post hoc tests $p>0.05$; all other comparisons $p>0.05$; Figures 4 and 7 ). Reduced herbivore damage on irLOX2 in 2011 could have increased the growth and reproduction of irLOX2 plants relative to WT, but cannot explain why irLOX2 plants instead displayed reduced growth and reproduction. Plants in 2011 were also damaged by flea beetles and grasshoppers $(<3 \%$ of canopy area, Kruskal-Wallis tests, $\mathrm{N}=24-28$, all comparisons $\mathrm{p}>0.05$, Figure 7 ).

We cannot exclude the possibility that reduced growth and reproduction of uninfested irLOX2 and hemi-irLOX2 plants in 2011 (Figure 6, statistics Table 3) might have been due to non-herbivory-related factors (e.g., differences in root health corresponding to GLV antimicrobial properties) which did not play a role in 2010. Because of this uncertainty, we conducted assay M4 (Figure 4) in which plants were carefully matched for size and prior reproduction (Figure 8), and this experiment is the more robust basis for our argument that GLV-mediated indirect defense increases plant reproduction.

\section{GLV-mediated Manduca spp. mortality positively correlates to plant reproduction}

To ensure that the correlated differences we observed in plant reproduction and $M$. sexta mortality were due to plant GLV emission and not to different timing and amounts of $M$. sexta damage, and to avoid the influence of non-herbivory-related factors, we conducted a Manduca spp. predation and plant performance assay during infestations M1 in 2010, and M4 in 2011 (Figure 4) for which all plants used were matched for size, as well as former damage and reproduction as necessary (Figure 8), and infested simultaneously with Manduca spp. neonates. We hypothesized that the $50 \%$ lower predation rates of Manduca spp. from GLV-deficient plants (Figure 5), combined with Manduca spp.'s negative effect on growth and reproduction (Figure 6), would result in reduced reproduction for 
Table 2. Numbers (N) of Geocoris spp. individuals (nymphs and adults) within $5 \mathrm{~cm}$ radii around plants used for predation experiments, counted within half an hour during the main period of Geocoris spp. activity.

\begin{tabular}{|c|c|c|c|c|c|c|c|}
\hline \multirow{2}{*}{$\begin{array}{l}\text { Experiment } \\
\text { Larval predation }\end{array}$} & \multirow{2}{*}{$\begin{array}{l}\text { Genotype } \\
\text { Dates }\end{array}$} & \multicolumn{3}{|c|}{ Geocoris spp. per day (n) } & & & \multirow[t]{2}{*}{ Plants (n) } \\
\hline & & May & 21 & 22 & & & \\
\hline \multirow[t]{5}{*}{ May 21-23, 2011} & WT & & 3 & 4 & & & 19 \\
\hline & ir $P l$ & & 6 & 6 & & & 24 \\
\hline & $\operatorname{irLOX2}$ & & 6 & 4 & & & 20 \\
\hline & hemi-irLOX2 & & 8 & 2 & & & 20 \\
\hline & Total & & 23 & 16 & & & 83 \\
\hline Egg predation & Dates & June & 3 & 4 & 5 & 7 & \\
\hline \multirow[t]{5}{*}{ June 2-6, 2011} & WT & & 2 & 5 & 2 & 1 & 18 \\
\hline & ir $P I$ & & 3 & 7 & 1 & 5 & 21 \\
\hline & $\operatorname{irLOX2}$ & & 4 & 2 & 0 & 3 & 21 \\
\hline & hemi-irLOX2 & & 1 & 1 & 2 & 2 & 24 \\
\hline & Total & & 10 & 15 & 5 & 11 & 84 \\
\hline
\end{tabular}

Numbers are shown as subtotals for each plant genotype and grand totals per day (in bold). DOI: 10.7554/eLife.00007.009

GLV-deficient versus matched GLV-producing plants if Geocoris spp. were present. Homozygous irLOX2 plants were excluded from these 'matched' experiments because they did not suffer reduced growth or reproduction from $M$. sexta feeding, and because they were too small in comparison to other lines in 2011 (Figure 6).

In both 2010 and 2011, we selected triplets of WT, irPI and hemi-irLOX2 plants similar in size, reproductive output, apparent health, and prior damage; damage from naturally occurring herbivores did not differ among these genotypes (Figure 7). In 2010, matched plants were part of infestation M1 (Figure 4) and thus it was not necessary to control for prior reproduction or M. sexta damage. Plants in 2010 received three lab strain $M$. sexta larvae per plant to a lower stem leaf. We recorded the mortality of $M$. sexta larvae and the reproductive output of plants until they began to set unripe seed. No reproductive meristems were removed, but flowers were removed and counted periodically over the first 10 days, as was done during infestation M3 in 2011 (Figures 4 and 6, statistics Table 3), to track plant reproduction while avoiding ripe seed capsules: the distribution of ripe seed is not permitted for genetically modified plants. In the absence of Geocoris spp. in 2010, genotypes did not differ in M. sexta mortality ( $\mathrm{N}=51$ larvae) - which in every observed case was due to a failure of the larva to feed-or plant reproduction ( $\mathrm{N}=17$ plants) (Figure 9). This, and the fact that flower production did not differ among genotypes in 2011 through infestation M3 despite flower removal (Figure 6), indicates that flower removal itself does not cause a difference among genotypes, and suggests that the other differences among genotypes in growth and reproduction seen in 2011 (Figure 6, statistics Table 3) are real.

In 2011, hemi-irLOX2, irPI and WT plants were matched prior to infestation M4 to exclude differences in growth, reproduction and Manduca spp. damage arising during M. sexta infestations M2 and M3 and from caged Manduca spp. during the egg predation assay (Figures 4, 7, and 8). Instead of regularly removing flowers, we removed all reproductive meristems from matched plants in 2011 by cutting inflorescences at their base. This allowed us to follow a new set of reproductive meristems through to seed set without incurring ripe seed. Because plants were matched prior to the assay, a similar number of reproductive meristems were cut from all plants, and thus all plants were similarly affected by this cutting (Figure 8 , see 'Discussion').

Because oviposition by native Manduca spp. moths provided sufficient eggs prior to the beginning of M4 (Figure 4), we decided to conduct this infestation with wild larvae and thereby demonstrate that native larvae, like larvae of the lab strain, are susceptible to GLV-mediated predation. To make M4 a realistic test, we placed one wild Manduca spp. neonate per plant on a lower stem leaf to mimic natural oviposition rates (Kessler and Baldwin, 2001). We again recorded the mortality of Manduca spp. larvae and the new reproductive output of plants until they began to set unripe seed. During the first 
A
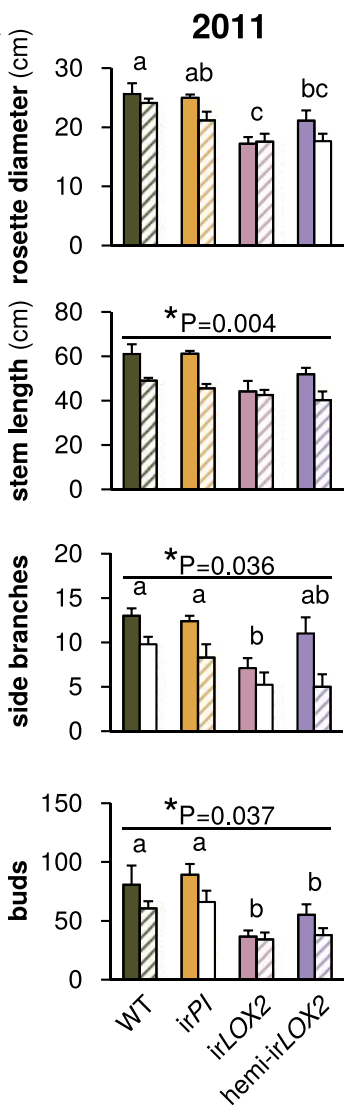

control

$\square M$. sexta* $^{*}$

2010
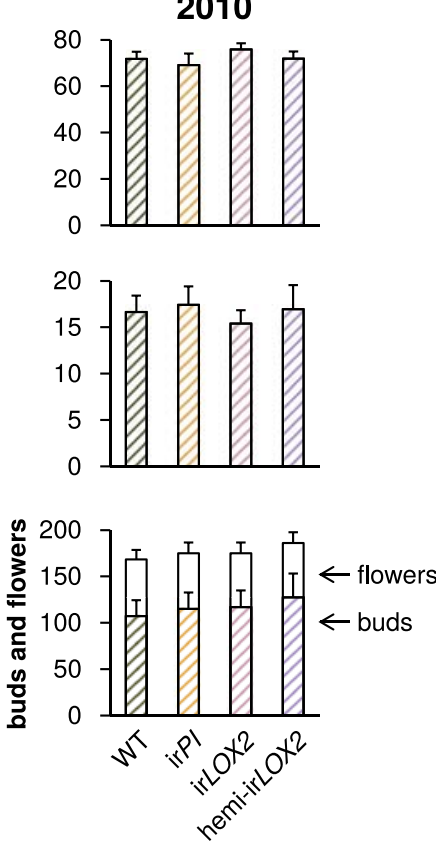

\section{B: 2011}

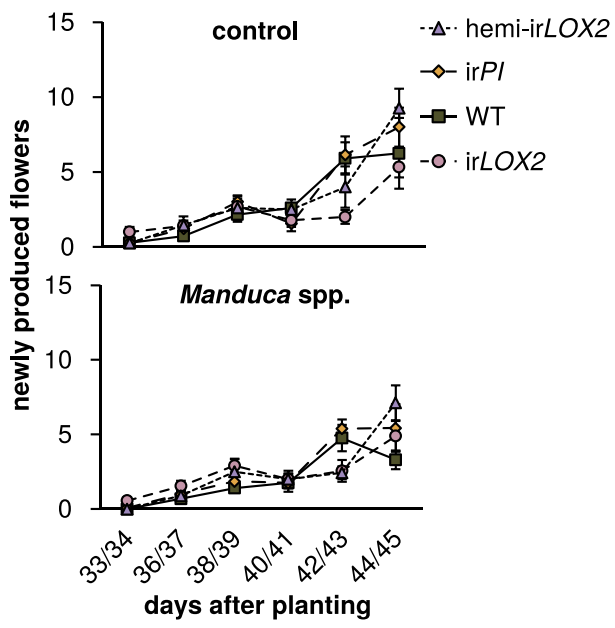

C: $\mathbf{2 0 1 0}$

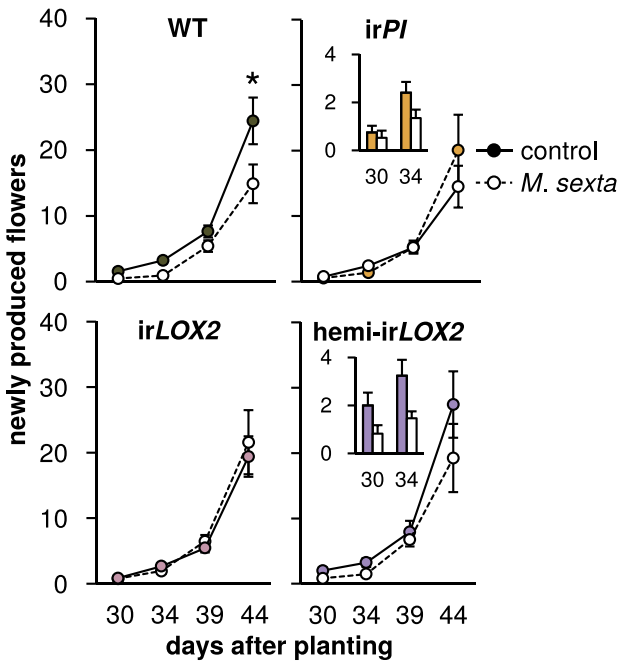

Figure 6. Growth and reproduction of plants during the 2010 and 2011 field seasons; graphs show means \pm SEM. (A) Final growth measurements for M. sexta-infested and uninfested control plants of each genotype in 2011 (left, 44-45 days after planting, N=11-17) or M. sexta-infested plants in 2010 (right, June 6, 2 days after the removal of fifth-instar M. sexta larvae). ${ }^{\star} p<0.05$ for Wilks' Lambda test of the effect of M. sexta feeding on growth and reproduction in 2011, day 44-45, in a two-way MANOVA with factors genotype and treatment $\left(F_{6,52}=2.287, p=0.049\right)$. ${ }^{*} p$-values above individual graphs denote the significance of M. sexta feeding over all genotypes in 2011 for the measurement shown in the MANOVA, or in a separate Mann-Whitney $U$-test for side branches (stem $F_{1,57}=9.155$; side branches, $U=270$; buds $F_{1,57}=4.572$ ); values for individual genotypes are in Table 3. ${ }^{a}, b, c$ Different letters denote significant $(p<0.05)$ differences between genotypes in 2011 for Scheffe post hoc tests (rosette diameter $F_{3,57}=8.791$, $p<0.001$, stem length $F_{3,57}=4.192, p=0.009$, number of buds $F_{3,57}=9.876, p<0.001$ ) or Bonferroni-corrected $p$-values for Mann-Whitney U-tests following a Kruskal-Wallis test (side branches $x^{2}=10.958$ ). In 2010, in the absence of Geocoris spp. activity, there were no significant differences between genotypes in the parameters shown with or without M. sexta infestation (Table 3). Bud numbers from 2010 are also shown in Figure 9. (B and C) Flower production for M. sextainfested and uninfested control plants from the beginning of flowering in (B) 2011 and (C) 2010. Flowers were counted and removed at the time points shown: each time point represents new flower production. Insets in (C) show the first two time points for irPI and hemi-irLOX2. ${ }^{*}<<0.05$ for the main effect of M. sexta infestation in a repeated-measures ANOVA with $\log _{2}$-transformed data (Table 3). Raw data for 2011 is in F6AB SchumanBarthelBaldwin2012growth_reproduction2011.xlsx and T4_SchumanBarthelBaldwin2012growth_reproduction2011.xlsx, and data for 2010 is in F6AC_SchumanBarthelBaldwin2012growth_reproduction2010.xlsx (Dryad: Schuman et al., 2012)

DOI: 10.7554/eLife.00007.010 
Table 3. Results of Mann-Whitney U-tests, Kruskal-Wallis tests, and ANOVAs for control vs M. sexta-infested plants of each genotype grown in the field in 2010 and 2011 (Figures 6 and 9)

\begin{tabular}{|c|c|c|c|c|c|c|c|c|c|c|c|c|c|c|c|c|}
\hline \multirow[t]{2}{*}{2010} & \multirow[b]{3}{*}{ Genotype } & \multicolumn{6}{|c|}{ Stem, buds, flowers } & & & & & & & & & \\
\hline & & \multicolumn{3}{|c|}{$\begin{array}{l}\text { Mann-Whitney, } \\
\text { Kruskal-Wallis }\end{array}$} & \multicolumn{3}{|c|}{ MANOVA, Wilks' lambda } & & & & & & & & & \\
\hline Comparison & & df & $x^{2}$ & $p^{*}$ & $d f$ & $\mathrm{~F}$ & $p$ & & & & & & & & & \\
\hline Treatment & All & 1 & 0.022 & 1.000 & 3,148 & 0.46 & 0.70 & & & & & & & & & \\
\hline \multirow[t]{2}{*}{ Genotype } & All & 3 & 2.909 & 0.802 & $29,360.344$ & 1.18 & 0.30 & & & & & & & & & \\
\hline & & \multicolumn{3}{|c|}{ Branches (n) } & \multicolumn{3}{|c|}{ Rosette diameter $(\mathrm{cm})$} & \multicolumn{3}{|c|}{ Stem length (cm) } & \multicolumn{3}{|c|}{ Buds (n) } & \multicolumn{3}{|c|}{ Flowers (n) } \\
\hline \multicolumn{2}{|l|}{2011} & \multicolumn{3}{|c|}{ Student's t-test } & \multicolumn{3}{|c|}{ Student's t-test } & \multicolumn{3}{|c|}{$\begin{array}{l}\text { MANOVA, Wilks' } \\
\text { lambda }\end{array}$} & \multicolumn{3}{|c|}{$\begin{array}{l}\text { MANOVA, Wilks' } \\
\text { lambda }\end{array}$} & \multicolumn{3}{|c|}{$\begin{array}{l}\text { MANOVA, Wilks' } \\
\text { lambda }\end{array}$} \\
\hline Comparison & Genotype & $d f$ & $\mathrm{t}$ & $\mathrm{p}$ & $d f$ & t & $\mathrm{p}$ & $d f$ & $\mathrm{~F}$ & $\mathrm{p}$ & $d f$ & $\mathrm{~F}$ & $\mathrm{p}$ & $d f$ & $\mathrm{~F}$ & $\mathrm{p}$ \\
\hline \multirow[t]{4}{*}{ Treatment $\times$ time } & WT & 26 & 1.696 & 0.102 & 26 & -0.870 & 0.932 & 5,22 & 3.871 & 0.011 & 5,22 & 3.188 & 0.026 & 3,24 & 1.213 & 0.326 \\
\hline & ir $P l$ & 26 & 1.024 & 0.315 & 26 & -0.161 & 0.873 & 5,22 & 0.991 & 0.446 & 5,22 & 0.656 & 0.660 & 5,22 & 0.525 & 0.755 \\
\hline & irLOX2 & 25 & 1.112 & 0.277 & 25 & -0.058 & 0.954 & 5,21 & 0.606 & 0.696 & 5,21 & 0.535 & 0.748 & 5,21 & 0.540 & 0.744 \\
\hline & hemi-irLOX2 & 22 & 1.753 & 0.094 & 22 & 1.140 & 0.267 & 5,18 & 1.118 & 0.386 & 5,18 & 3.001 & 0.038 & 4,19 & 0.723 & 0.587 \\
\hline
\end{tabular}

2010: Numbers of side branches (Mann-Whitney, Kruskal-Wallis), stem length, and final numbers of buds and flowers (MANOVA) were recorded in a single measurement at the end of M1 (Figure 4). Numbers of newly produced flowers were counted repeatedly upon flower removal, and Wilks' Lambda F values for the main effect of $M$. sexta feeding are shown from repeated-measures ANOVAs across all measurements; Wilks' $F$ values for the M. sexta-by-time interaction were not significant. *Bonferroni-corrected p-values.

2011: Because many plants had few or no side branches before the final measurement, and rosette diameters did not change over the period that plants were measured, t-tests are shown for the final measurement of these parameters in M3 (Figure 4). For stem lengths, numbers ( $\mathrm{n}$ ) of buds, and numbers of flowers, Wilks' lambda F values for the M. sexta-by-time interaction are shown from repeated-measures ANOVAs across all measurements. Significant p-values are given in bold.

DOI: 10.7554/eLife.00007.011

to third larval instars in which larvae are vulnerable to Geocoris spp. predation (Kessler and Baldwin, 2001), wild Manduca spp. mortality was $38 \%$ on hemi-irLOX2 plants vs $62-76 \%$ on matched WT and irPI plants; the overall mortality of larvae on all three lines was significantly different $(\mathrm{N}=21$ larvae, Bonferroni-corrected pairwise comparisons by Friedman tests, $\mathrm{p}<0.01$ ) (Figure 9). Although Manduca spp. mortality on hemi-irLOX2 jumped to $70 \%$ in the fourth and semi-final larval instar, this was likely due to predation by whiptail lizards (Cnemidophorus spp.) which were present on the field plot: these lizards predate late-instar Manduca and are attracted to short-chain fatty-acid volatiles produced by the larvae due to ingestion of acyl sugars in plant trichomes (Stork et al., 2011; Weinhold and Baldwin, 2011).

The plants used in M4 had not previously differed in their reproduction except that hemi-irLOX2 plants had produced more flowers than WT, but not irPI plants (Figure 8). By the end of the assay, the hemi-irLOX2 plants had produced $40-50 \%$ fewer buds and flowers than matched WT and irPI plants ( $N=21$ plants, $\mathrm{p}<0.05$ in Scheffe post hoc tests for hemi-irLOX2 vs WT and irPI flowers and buds following a repeated-measures MANOVA over all flower and bud counts, Wilks' Lambda for the interaction of line and day: $F_{12,110}=2.835, p=0.002$ ) (Figure 9). This reduced bud and flower production was not due to accelerated seed set: unripe seed capsules on hemi-irLOX2 plants were also reduced by $50 \%$ ( $N=21$ plants, $p=0.021$ for hemi-irLOX2 vs irPI in a Scheffe post hoc test following an ANOVA with genotype as the factor, $F_{2,60}=4.142, p=0.021$ ) (Figure 9). These data demonstrate that herbivore-induced GLV emissions function as indirect defenses by increasing predation of Manduca spp. larvae twofold, resulting in a twofold increase in bud and flower production for $N$. attenuata in its native habitat.

\section{M. sexta and M. quinquemaculata perform similarly on plants}

To ensure that our results were not biased by the use of wild Manduca spp. larvae, which comprised both $M$. sexta and $M$. quinquemaculata, we analyzed the growth (length over time) and instar change of larvae on plants in M4 by larval species. M. sexta and M. quinquemaculata did not differ in their growth or instar progression ( $\mathrm{N}=11-13$, repeated measures ANOVA for days 4-11, Wilks' Lambda for 


\section{A: 2010}

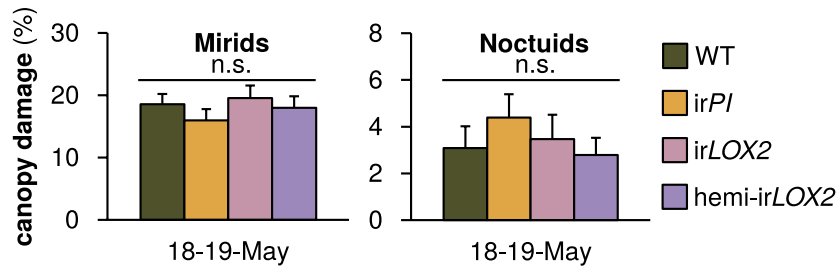

\section{B: 2011}

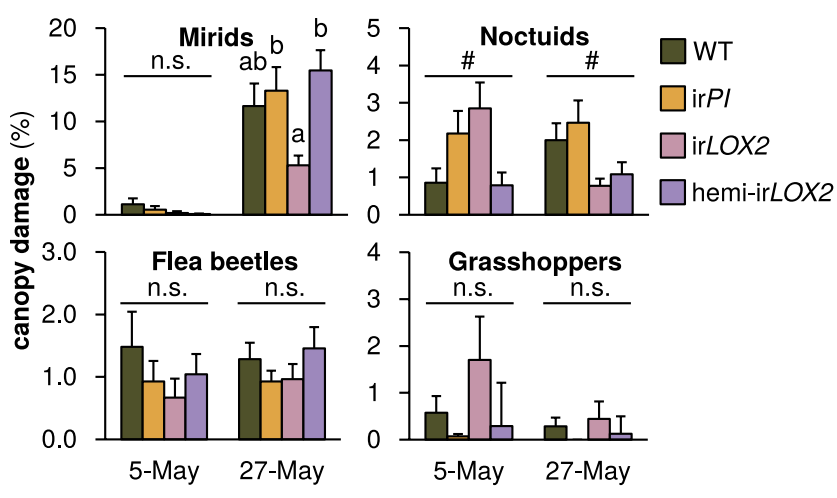

\section{C: 2011}
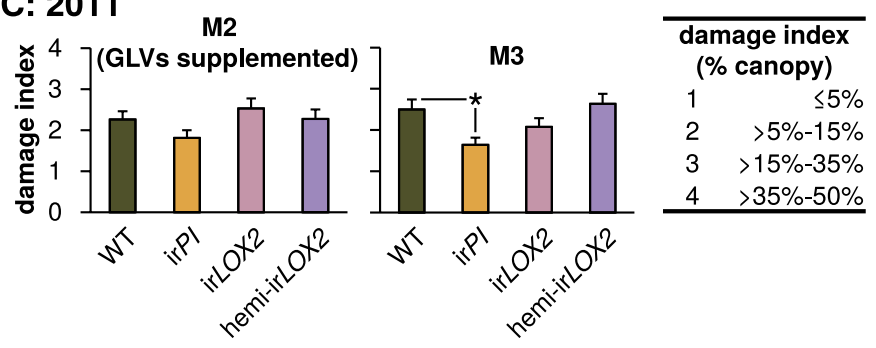

Figure 7. Herbivore damage to plants during the 2010 and 2011 field seasons (means+SEM). For a timeline of Manduca infestations M1-M4, see Figure 4A. (A) Total canopy damage due to naturally occurring herbivores before the start of infestation M1 in 2010, N=17. For raw data, see F7A_SchumanBarthelBaldwin2012herbivoreDamage2010. xlsx (Dryad: Schuman et al., 2012). (B) Total canopy damage due to naturally occurring herbivores before infestation M2 (May 5) and near the end of M3 (May 27) in 2011, N=24-28. a,b Different letters denote significant $(p<0.05)$ differences between genotypes in Scheffe post hoc tests following one-way ANOVAs for arcsine-transformed data at each timepoint (mirids May $27 F_{3,103}=5.291, p=0.002$; noctuids May $27 F_{3,103}=3.503, p=0.018$ ); n.s.: not significantly different. $\# \mathrm{p}<0.05$ for the main effect of genotype on noctuid damage in a Bonferroni-corrected Kruskal-Wallis test, May 5 ( $\left.X^{2}=11.239, p=0.027\right)$. (C) Damage in 2011 from M. sexta larvae used in the predation assays in M2 (left panel) and M3 (right panel). GLVs were externally supplemented to plants in infestation M2 and not in M3. Total canopy damage was estimated, using the index, by an independent observer without knowledge of plant identity $(N=11-17) .{ }^{*} p<0.05$ in a Mann-Whitney $U$-test between irPl and WT on May $28(U=54, p=0.046)$; the difference on May 15 was not significant $(p>0.1)$. Note that scales differ. Raw data for $(B)$ and $(C)$ is in F7BC_ SchumanBarthelBaldwin2012herbivoreDamage2011.xlsx (Dryad: Schuman et al., 2012). DOI: 10.7554/eLife.00007.012

the interaction of day and species: $F_{11,12}=1.356, p=0.311$ ). Because larvae of the two species cannot be distinguished before the third instar, we could not test whether mortality was equal for both species in the first three instars; however, because other collections of wild eggs around the same time as the collection for our experiment yielded a 1:1 ratio of species, and because our ratio of the species remained 1:1 after larvae reached the third instar, it is likely that mortality of the two species was equal prior to the third instar. 
A
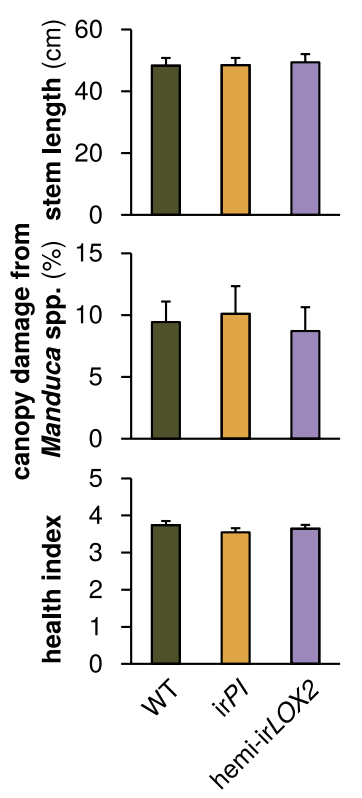

B

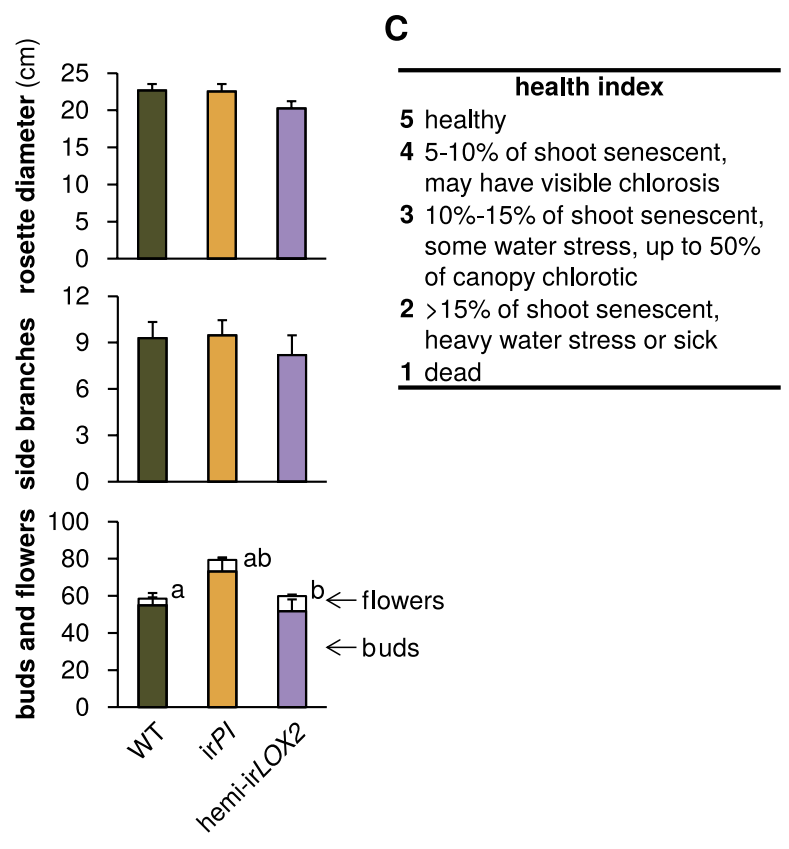

Figure 8. Comparison of plants used in triplets for infestation M4 in 2011 (see Figure 4A); graphs show means+SEM ( $N=21$ plants). (A) Parameters used to match plants in triplets. Measurements and assessments are from the first day of M4. (B) Final measurement of prior growth and reproduction for plants used for triplets; data are from the final two measurements during infestation M3 (see Figure 4A). ${ }^{a, b}$ Different letters denote significant differences $(p<0.001)$ for flower number in Scheffe post hoc tests following a MANOVA with all measurements and genotype as the factor $\left(F_{2,60}=8.668, p<0.001\right)$. (C) Health index used in (A). For raw data, see F8_SchumanBarthelBaldwin2012triplets.xlsx (Dryad: Schuman et al., 2012). DOI: 10.7554/eLife.00007.013

\section{Manduca spp. response to mock predator attack is altered by TPI consumption}

TPls had a less consistent and, contrary to our expectations, negative effect on Manduca spp. predation (Figure 5); furthermore, there was no positive effect of TPIs on plant growth and reproduction (Figure 6, statistics Table 3) and only a marginal effect of TPIs on Manduca spp. growth under natural conditions ( $N=13-26$ second instar larvae during $M 2$, one-way ANOVAs with genotype as the factor, $F_{3,77}=2.792, p=0.046$, all post hoc tests $p>0.05$, Figure $10 ; N=8$ second instar larvae during M4, paired t-test between matched WT and irPI, $p=0.052$ ). We hypothesized that the reduced access to protein for larvae feeding on TPI-producing plants might nevertheless affect Manduca spp. behavior independently of larval size. Indeed, wild Manduca spp. larvae feeding on WT plants (infestation M4, Figure 4) reacted more sluggishly to experimental provocation than size-matched larvae on irPI plants: they were $75 \%$ less likely to attack when lifted off of the leaf ( $N=5$ second-instar larvae matched for size, $p=0.035$ in paired t-test) (Figure 10, Videos 1 and 2).

We were careful not to harm wild larvae so that we could monitor their natural mortality and consequences for plant reproduction (Figure 9). To more accurately imitate Geocoris spp. attack, we developed an off-plant assay with larvae from the laboratory M. sexta strain feeding on detached leaves from field-grown plants, in which size-matched larvae were poked, pierced and lifted using an insect pin to mimic the Geocoris spp. beak (Figure 10, Videos 3 and 4). Similarly to the on-plant assay, larvae fed on WT leaves were $50 \%$ less likely to successfully attack the insect pin, either when initially poked, or poked and lifted with the pin ( $N=20$ first-instar larvae matched for size, $\mathrm{p}<0.05$ in paired t-tests) (Figure 10). We also monitored recovery post-trial and found that WT-fed larvae ceased to grow for at least $24 \mathrm{hr}$ after simulated attack, while irPl-fed larvae 


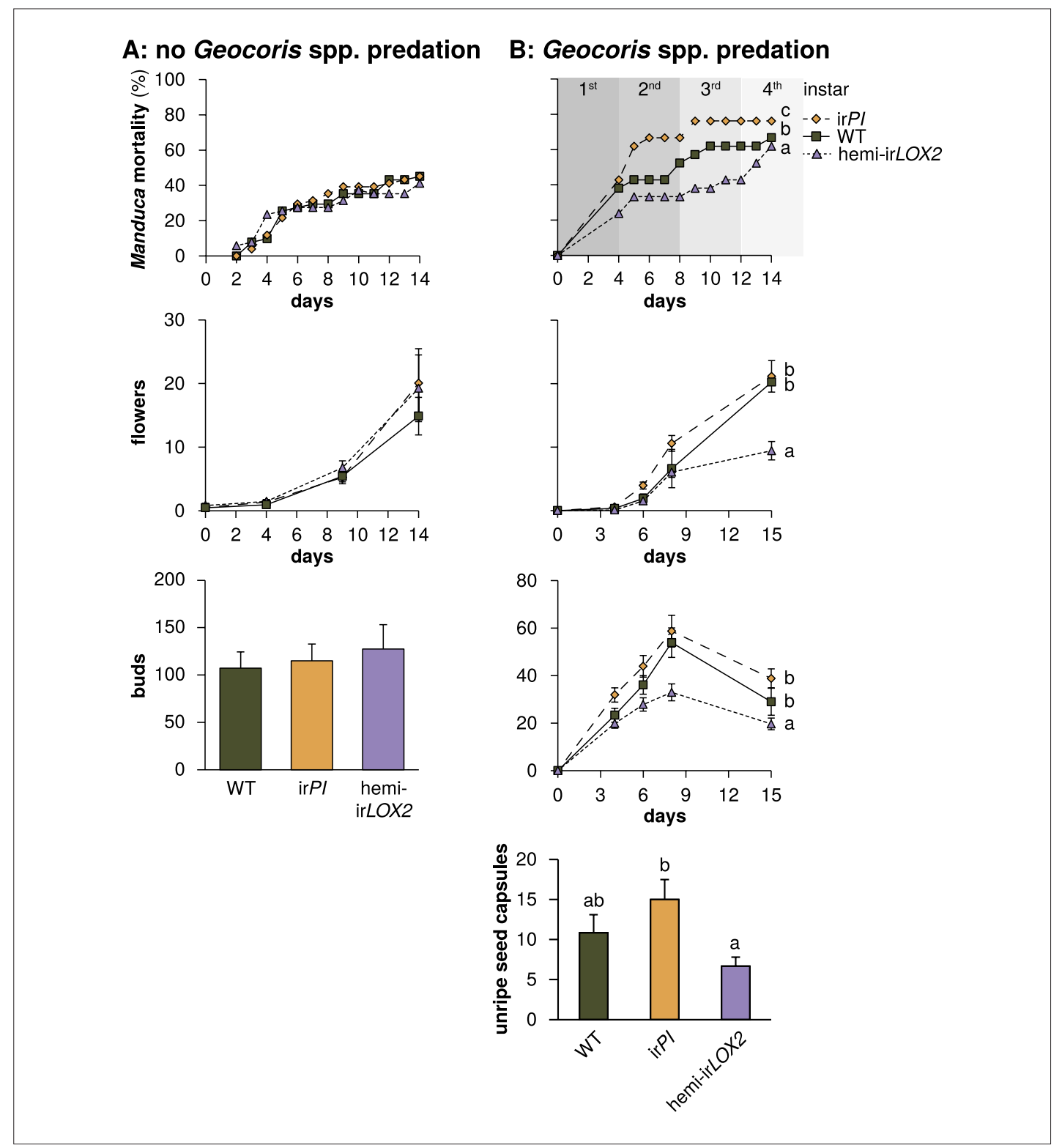

Figure 9. Cumulative mortality of Manduca spp. larvae and numbers of reproductive units produced by infested plants in 2010, in the absence of Geocoris spp. predation, and in 2011, when Geocoris spp. were active predators of Manduca spp. (A) In 2010, flowering plants matched for size ( $N=17)$ were each infested with three M. sexta neonates from a laboratory culture ( $\mathrm{N}=51$ larvae), which were allowed to reach the final instar on plants. The upper panel shows larva mortality over time, which reached a maximum of $40 \%$ by the fifth instar, after 12 days. Flower production (lower panel) did not differ, nor did any other parameters of plant size and reproduction (Figure $\mathbf{6}$, Table 3) including number of buds produced by June 6, which was day 19 after infestation and day 49 after planting in the field. For raw data, see F9A_SchumanBarthelBaldwin2012data2010.xlsx (Dryad: Schuman et al., 2012). (B) In 2011, plants ( $N=21)$ were matched for size, prior reproduction, health, and previous damage by Manduca spp. and other herbivores (Figures $\mathbf{7}$ and 8) following the end of infestation M3 (Figure 4), and reproductive meristems were removed. Matched plants were infested with one wild Manduca spp. neonate each (M4 in Figure 4), and Manduca spp. larvae were allowed to reach the fourth (penultimate) instar. Larval mortality (upper panel) reached a maximum of 76\% after larvae transitioned from the second to third instar (days 9 and 10), at which time larval mortality on hemi-irLOX2 was only half as great as on WT or irPl; larvae beyond this stage are not susceptible to Geocoris spp. (Kessler and Baldwin, 2001). Flower and bud production (lower panel) was twice as great in WT and irPl as in hemi-irLOX2, and numbers of flowers and buds correspond to numbers of seed capsules: hemi-irLOX2 plants also produced fewer unripe seed capsules than WT or irPI plants. For raw data, see F9B_SchumanBarthelBaldwin2012data2011.xlsx (Dryad: Schuman et al., 2012). a, b, c Different letters indicate Figure 9. Continued on next page 
Figure 9. Continued

significant differences $(p<0.01)$ in Bonferroni-corrected pairwise Friedman tests (Manduca spp. mortality), or Scheffe post hoc tests of hemi-irLOX2 versus WT and irPI flowers and buds following a repeated-measures MANOVA over all flower and bud counts shown (results of Greenhouse-Geisser-corrected univariate tests for the interaction of line and day: buds, $F_{4.988,149.653}=5.297, p<0.001$; flowers, $F_{3.722,111.657}=4.403, p=0.003$ ), or significant differences $(p<0.05)$ in Scheffe post hoc tests following an ANOVA for unripe seed capsules at day 15 with genotype as the factor $\left(F_{2,60}=4.142, P=0.021\right)$.

DOI: 10.7554/eLife.00007.014

continued to grow ( $p=0.016$ in Student's t-test); mortality did not differ $(p=0.527$ in Fisher's exact test) (Figure 10).

Thus TPIs did not increase plant reproduction under attack from Manduca spp. in nature, but may support indirect defense by weakening the response of larvae to predator attack. The contradictory higher predation rates of Manduca spp. larvae from irPI than from WT plants (Figure 5) might reflect Geocoris spp.'s feeding preference, if irPl-fed larvae are more nutritious than WT-fed larvae (Kaplan and Thaler, 2011).

\section{Discussion}

Our data demonstrate that herbivore-induced GLV emissions function as indirect defenses by increasing predation of Manduca spp. twofold, resulting in a twofold increase in bud and flower production for $N$. attenuata in its native habitat. In contrast, there was no positive effect of TPls on plant growth and reproduction and no significant effect of TPIs on Manduca spp. growth under natural conditions; however, TPIs may support indirect defense by weakening the response of larvae to predator attack. Although this indicates that predation rates from irPI plants should be reduced, we observed a tendency towards higher predation rates from irPI than from WT plants; this could reflect Geocoris spp.'s preference if irPl-fed larvae are more nutritious than WT-fed larvae (Kaplan and Thaler, 2011).

\section{WT levels of TPIs in hemi-irLOX2 plants are likely due to gene dosage effects}

The hemi-irLOX2 plants used in this study were created by crossing homozygous irPI and irLOX2 plants, but the irPI construct was not active in the hemizygous state (Figure 1). We continued to use this cross for its less severely reduced GLV production in comparison to homozygous irLOX2 plants (Figures 2 and 3), which likely permitted growth and reproduction comparable to irPI and WT in 2011 that was essential for plant matching prior to the final assays of Manduca spp. mortality and plant reproduction (M4, Figures 4 and 9). It is common molecular biology knowledge that functional RNAi constructs may be rendered ineffective as a result of insufficient gene dosage, for example, Travella et al. (2006) and references therein, which may occur when an RNAi construct is present in the hemizygous state (García-Pérez et al., 2004). The 35 S promoter which drives the transcription of the RNAi construct may also be methylated: an epigenetic effect which can reduce the dose of RNAi in individual plants within a single transformed line (A Weinhold, unpublished data). This may have occurred in the irPI parent used for the creation of the hemi-irLOX2 line, although loss of activity of the irPl construct was not observed in the homozygous irPI line over the lifetime of plants in the field (Figure 1).

\section{Our best measures of reproduction for transgenic plants in the field demonstrate the positive effect of GLV-mediated indirect defense}

Although the production of viable offspring is the accepted definition of Darwinian fitness, we are not permitted to allow transgenic plants to disperse ripe seed in the field, and measures to prevent seed dispersal, such as bagging meristems, strongly affect production of buds and flowers and can also affect seed viability by increasing temperature, and decreasing respiration and photosynthesis of reproductive tissue and associated green tissue.

For field-grown N. attenuata plants, fewer than $5 \%$ of buds and flowers (in total) are aborted by healthy (not diseased) plants, and abortion seems always to be due to damage by insects (M C Schuman and I T Baldwin, personal observation, June 2010). Plants are self-compatible and more than $70 \%$ of seed set from plants in native populations results from fertilization via self-pollen 


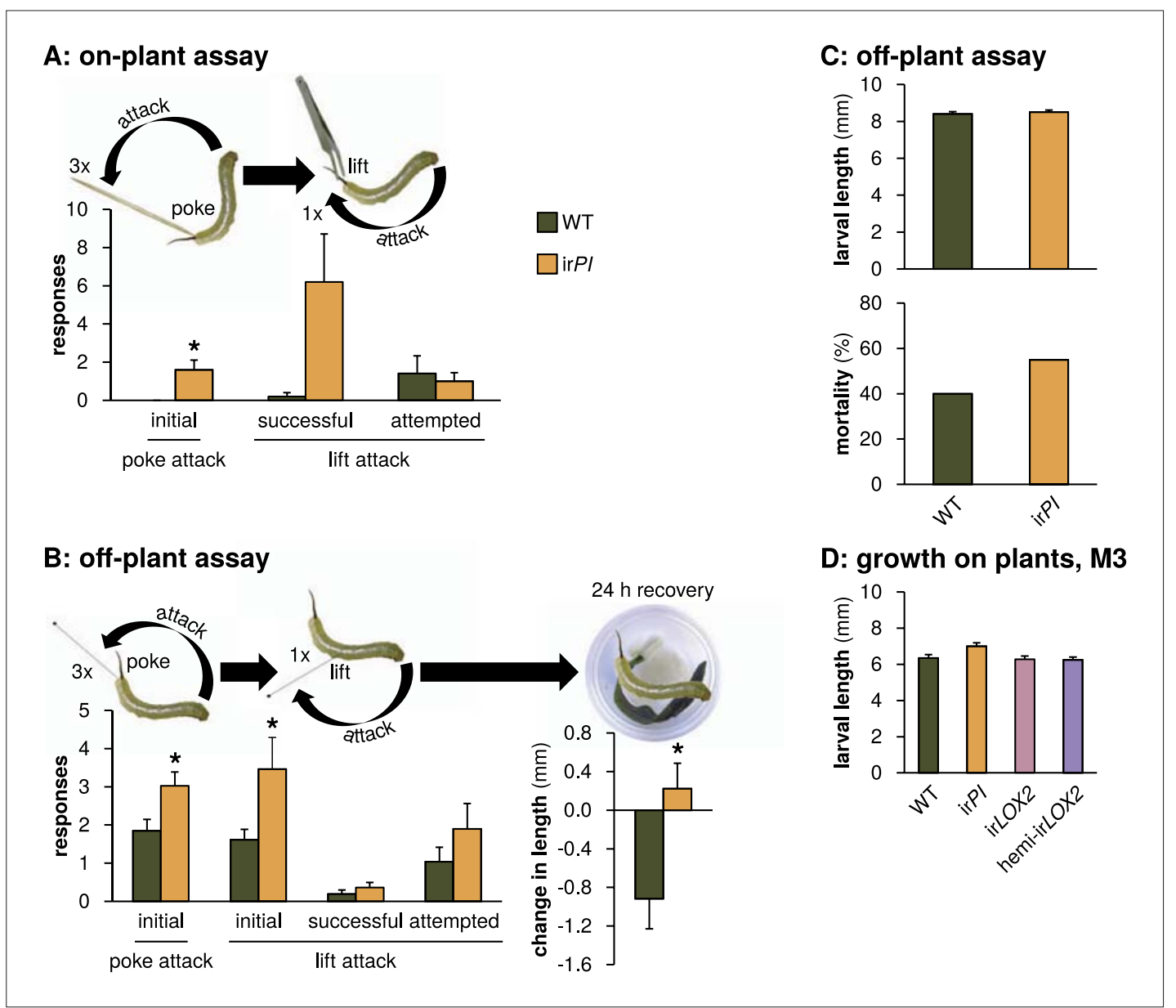

Figure 10. Mock Geocoris spp. predation assays with Manduca spp. larvae fed on WT or irPI plants. (A) Response of wild Manduca spp. (Figure 4A) on plants in the field to poking with a toothpick and lifting with a featherweight forceps ( $\mathrm{N}=5$ second-instar larvae matched for size). We first poked larvae below the horn three times, $3 \mathrm{~s}$ apart, with the end of a toothpick and counted how often they attacked the toothpick, defined as the larva whipping its head around toward the toothpick and making contact. We then lifted larvae from the plant using the forceps and counted how often they attempted to attack, or succeeded in attacking the forceps over $15 \mathrm{~s}$. In an attempted attack, the larvae moved from hanging at a $180^{\circ}$ angle below the forceps vertically toward the forceps; and in a successful attack, the front end of the larva made contact with the forceps, before returning to its original position. All individuals were recorded and responses were counted from videos (see Videos 1 and 2). ${ }^{*} p<0.05$ in a paired t-test. (B) Left, response of M. sexta from a laboratory strain raised for $48 \mathrm{hr}$ in boxes on either WT or irPl leaf tissue ( $\mathrm{N}=20$ first-instar larvae matched for size) to being poked, pierced and lifted with an insect pin. Right, growth of larvae in the following $24 \mathrm{hr}$. The procedure was identical to that for the on-plant assay described above, except that larvae were poked with an insect pin rather than a toothpick, and then pierced in the rear flank and lifted with the same insect pin (see Videos 3 and 4). ${ }^{*} p<0.05$ in a paired t-test. The length of each larva was measured prior to poking and lifting. Afterward, larvae were placed in individual cups, each with a moist paper towel round and fresh WT or irPl leaf tissue, and length of the larvae in millimeters was again measured after $24 \mathrm{hr}$; mortality did not differ between WT- and irPl-fed larvae. ${ }^{*} \mathrm{p}<0.05$ in a Student's t-test. (C) Upper panel, length of first instar larvae fed for 2 days on WT or irPI tissue and size-matched for use in the off-plant behavioral assay mimicking Geocoris attack (B); lower panel, mortality of first instar larvae $24 \mathrm{hr}$ after mock Geocoris attack as described in (B). Mortality was not significantly different in a Fisher's exact test. (D) Larval length in the first instar after 2 days on plants in the field: larvae on irPl were not significantly larger. Length of surviving larvae was measured in a predation assay during infestation M3 (Figures 4 and 5C), N=13-26 larvae. Length was not significantly different for larvae feeding on irPI in a one-way ANOVA with genotype as the factor $\left(F_{3,77}=2.792, p=0.046\right.$, all post-hoc tests $\left.p>0.05\right)$. For raw data, see F10_SchumanBarthelBaldwin2012Manduca.xlsx (Dryad: Schuman et al., 2012).

DOI: 10.7554/eLife.00007.015 
(Sime and Baldwin, 2003). Thus numbers of buds and flowers correlate to lifetime seed capsule production, which in turn correlates to lifetime seed production, which has been used as a proxy measure of Darwinian fitness (Baldwin, 1998; van Loon et al., 2000; Hoballah and Turlings, 2001). The transgenic lines used do not vary in seed mass or their seedling viability under laboratory conditions.

\section{Meristem removal from plants before infestation M4 was necessary despite matching, and affected all plants similarly}

During the 2011 experiment, we saw a large and reproducible difference in predation from GLVemitting versus GLV-deficient plant genotypes, which was not observed in 2010 due to an absence of Geocoris spp. predators in that year. This difference in predation rate correlated to a difference in plant growth and reproduction which was also not observed in 2010. To rigorously test the consequences of GLV-mediated predation of Manduca spp. on plant reproduction, we selected triplets of WT, ir PI and hemi-irLOX2 plants similar in size, previous reproductive output, apparent health, and prior damage to carry out Manduca spp. mortality and plant reproduction assays (M1 in 2010, M4 in 2011, Figure 4). We removed all reproductive meristems from matched plants in 2011 to allow us to follow plant reproduction over full Manduca spp. larval development without incurring ripe transgenic seed capsules. In 2010 (and during infestation M3 in 2011, Figure 4), we had removed and counted flowers regularly to track reproduction while avoiding ripe seed; this did not cause a difference in reproduction among genotypes (Figure 6), but we elected to avoid flower removal during infestation M4 by removing reproductive meristems prior to the beginning of the assay.

The hemi-irLOX2 plants chosen in 2011 had produced more flowers than WT - but not more than irPl-prior to the start of infestation M4 (Figures 4 and 8). This did not correspond to more cuts on average for hemi-irLOX2 when removing reproductive meristems: meristems were cut at the bases of inflorescences which contained mostly buds, and the number of these did not differ for the plants chosen, nor did the number of side branches (Figure 8) which bore most reproductive meristems. Therefore, in the absence of additional effects during infestation M4, the reproduction of the matched hemi-irLOX2 plants should have been similar to that of WT and irPI.

\section{Conclusions and outlook}

By indicating the long-sought indirect defensive function of HIPVs, these data set the stage for the use of HIPVs as part of integrated pest management strategies (IPM), which rely in part on recruiting biological control agents to reduce pesticide use (Horne and Page, 2008). These agents are usually naturally occurring generalist parasitoids and predators, such as Geocoris spp. (Eubanks and Denno, 1999, 2000; Allison and Hare, 2009; Allmann and Baldwin, 2010). HIPVs are produced by genotypes of most, if not all crop plants and IPM would benefit from selective breeding or engineering of HIPV emission (Kos et alo, 2009) rather than relying on alternatives such as controlled release dispensers, which have mixed success and require large amounts of synthetic HIPVs (Kaplan, 2012). Pls may be employed to enhance the efficiency of indirect defense, especially combined with toxins like Bt that directly target herbivores and are safe for biological control agents. With growing concerns about field-evolved Bt resistance (Liu et alo, 2010), indirect defenses promise an effective 'first line of defense' against agricultural pests, to which not even specialist herbivores are likely to rapidly evolve resistance.

\section{Materials and methods}

\section{Plants, growth conditions and field plantations}

Seed germination, glasshouse growth conditions, and the Agrobacterium tumefaciens (strain LBA 4404)-mediated transformation procedure have been described previously (Krügel et al., 2002). Seeds of the 31st generation of the inbred 'UT' line of Nicotiana attenuata (Torr. ex S. Wats.) were used as the wild-type plant in all experiments. For the field experiment, seedlings were transferred to $50 \mathrm{~mm}$ peat pellets (Jiffy) 15 days after germination and gradually hardened to the environmental conditions of high sunlight and low relative humidity over 10 days. Small, adapted, size-matched rosette-stage plants were transplanted into a field plot in a native habitat in Utah and watered thoroughly once at planting and as needed over the first 2 weeks until roots were established; all plants received the same watering regime in each year. WT, irPI, irLOX2 and hemi-irLOX2 plants were arranged in quadruplets $(\mathrm{N}=40-50)$ of one plant per genotype, with individuals $0.5 \mathrm{~m}$ 


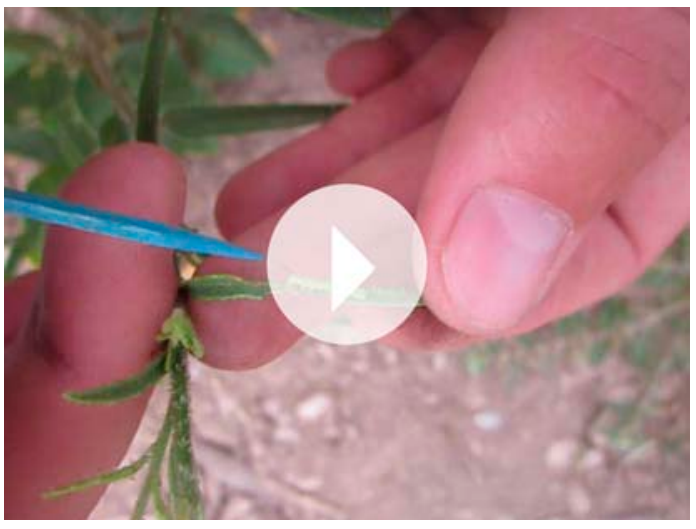

Video 1. On-plant assay, plant 7u, WT, June 18, 2011. DOI:10.7554/eLife.00007.016 apart, a distance sufficient to allow predators and herbivores to distinguish volatiles from neighboring plants (Kessler and Baldwin, 2001). Quadruplets were arranged so that no two adjacent plants were of the same genotype (Figure 4). In 2010, the field plot was a first-year plot located at latitude 37.141, longitude 114.027; in 2011, plants were planted at a second, older field site across a river from the first, located at latitude 37.146, longitude 114.020. Field plantations were conducted under APHIS permission numbers 06-242-3r-a3 (2010 and 2011) and 10-349-102r (2011).

We used previously characterized, homozygous, inverted-repeat (ir) RNAi transformed lines of the second transformed generation $\left(T_{2}\right)$ to silence GLV biosynthesis: irLOX2 line number A-04-52-2 (Allmann et al., 2010), and TPI activity: irPI line number A-04-186-1 (Steppuhn and Baldwin, 2007). Vector construction and the pSOL3 plasmid have been described previously (Bubner et al., 2006). A cross was created between irLOX2 and irPI homozygous lines; however, the hemizygous irPI construct did not silence TPI activity or transcripts, and these plants therefor served as vector controls for comparison with irPl and had slightly greater residual GLV production than irLOX2 (see 'Results'). They are thus referred to as hemizygous (hemi-) irLOX2 plants.

\section{Manduca spp. eggs and larvae}

Wild Manduca spp. eggs were collected for field assays when available from natural ovipositions. $M$. sexta and M. quinquemaculata (hereafter Manduca spp.) were both ovipositing at the time experiments were conducted; the species of larvae was identified at the third instar and recorded (earlier instars of these two species cannot be distinguished morphologically). M. sexta and M. quinquemaculata oral secretions (OS) are highly similar in their composition (Halitschke et al., 2001) and elicit similar volatiles (Halitschke et al., 2001; Kessler and Baldwin, 2001) and defense genes (Schittko et al., 2001) in N. attenuata. Eggs from laboratory-reared M. sexta, kindly provided by Dr. Carol Miles at SUNY Binghampton, were used in the field when wild Manduca spp. eggs were not sufficiently abundant. Eggs were allowed to hatch in well-aerated boxes on fresh $N$. attenuata leaf tissue over a moistened paper towel. M. sexta larvae used to elicit glasshouse-grown plants, or to collect oral secretions (OS) for plant treatments, were taken from an in-house colony at the Max Planck Institute for Chemical Ecology in Jena, Germany.

\section{Manduca spp. infestation and W+OS treatment of plants}

Because GLVs influence Manduca spp. oviposition (De Moraes et al., 2001; Kessler and Baldwin, 2001; Fraser et al., 2003), and the timing and extent of Manduca spp. oviposition varies from year to year, we created even, synchronous oviposition events by infesting plants with Manduca spp. larvae, either from a lab-reared culture or from wild collections (see Manduca spp. eggs and larvae). Larvae used for plant infestations were placed as neonates on a rosette or lower stem leaf at a standardized position for each assay, and monitored mornings and evenings, during times outside of the main period of Geocoris spp. activity that occurs at midday. Plants in field experiments were either infested with Manduca spp. larvae as described above, or left uninfested (control). There were four infestations over both years of the experiment, denoted M1-M4 in Figure 4.

For measuring headspace GLVs in the field and for glasshouse assays, plants were treated with wounding and $M$. sexta OS (W+OS) as a standardized method to mimic Manduca spp. feeding. Pure OS collected from fourth to fifth instar M. sexta larvae from the Jena colony fed on WT plants was diluted 1:5 with distilled water before use; even 1000-fold diluted OS is still sufficient to cause most OS-elicited responses (Schittko et al., 2000). For field-grown plants, a similar, mature, non-senescent leaf was chosen from each plant; for glasshouse-grown plants, the two adjacent older leaves (nodes $+1,+2$ ) to the leaf undergoing a source-sink transition (node 0 ) on rosette-stage plants were used for $\mathrm{Pl}$ and LOX2 transcript quantification, and the +2 node of a separate set of bolting plants was used for 


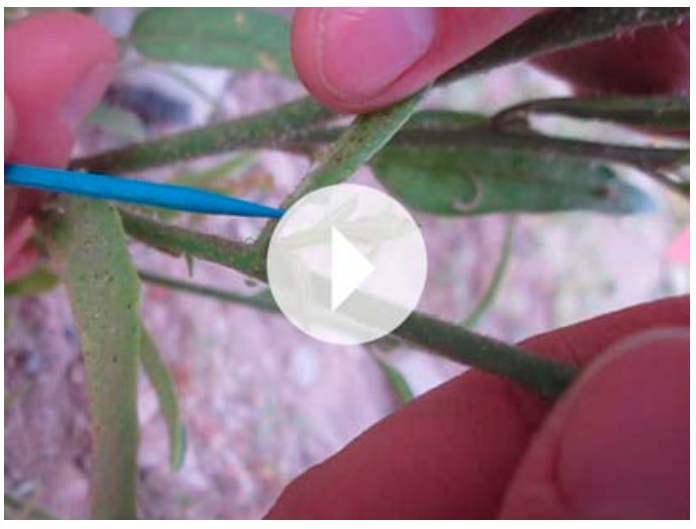

Video 2. On-plant assay, plant 2o, irPI, June 18, 2011. DOI:10.7554/eLife.00007.017 measuring headspace volatiles. The leaf chosen for treatment was wounded by using a fabric pattern wheel run over the adaxial surface to make six rows of holes in the lamina, three rows on either side of the midvein. $20 \mu \mathrm{L}$ of 1:5 diluted OS were deposited on the adaxial surface and gently rubbed over the holes with a gloved finger. Control plants were left untreated.

\section{Plant tissue harvests and sample handling}

For field-grown plants and glasshouse-grown $M$. sexta-fed plants, a similar, mature, non-senescent systemic (undamaged) leaf was chosen from each plant; for glasshouse-grown plants used to measure $P I$ and LOX2 transcripts, the leaves at nodes +1 and +2 (treated leaf positions) were harvested. Leaves were cut at the petiole and wrapped in a double layer of aluminum foil. In the field, harvested leaves were immediately frozen on dry ice insulated with ice packs frozen at $-20^{\circ} \mathrm{C}$; samples were stored at $-20^{\circ} \mathrm{C}$ until transport to Jena on dry ice, where they were kept at $-80^{\circ} \mathrm{C}$ until analysis. Leaves harvested from glasshouse-grown plants were flash-frozen in liquid nitrogen and kept at $-80^{\circ} \mathrm{C}$ until analysis. All sample processing was carried out over liquid nitrogen until the addition of the extraction solvents. Prior to analysis, entire leaves were ground with a mortar and pestle and transferred to a $2 \mathrm{~mL}$ microcentrifuge tube for storage. For specific measurements, aliquots were weighed into microcentrifuge tubes containing two steel balls and finely ground in a GenoGrinder (SPEX Certi Prep) prior to extraction.

\section{Quantification of TPI activity}

TPI activity was quantified in $100 \mathrm{mg}$ of tissue from systemic leaves on Manduca spp.-infested plants using a radial diffusion assay as previously described (van Dam et al., 2001).

\section{Quantification of PI and LOX2 transcripts}

Leaf samples were from control plants or plants treated with $\mathrm{W}+\mathrm{OS}$. Treated leaf positions were harvested at the peak of transcript accumulation for $\mathrm{Pl}, 12 \mathrm{hr}$ (Wu et al., 2006) and LOX2, $14 \mathrm{hr}$ (Allmann et al., 2010). Total RNA was extracted from leaves using the TRlzol reagent (Invitrogen), and a $0.5 \mu \mathrm{g}$ aliquot of total RNA of each sample was reverse-transcribed using oligo(dT) 18 and RevertAid H Minus reverse transcriptase (Fermentas) following the manufacturer's instructions. Quantitative real-time PCR (qPCR) was performed with a Mx3005P Multiplex qPCR system (Stratagene) and the qPCR Core kit for SYBR Green I (Eurogentec). Transcripts were quantified using external standard curves for each gene. Elongation factor $1 A$ (EF1A) transcript abundance in each sample was used to normalize total cDNA concentration variations. Samples of RNA used to make cDNA were pooled to the same dilution as in cDNA samples and run alongside cDNA in all qPCRs to control for gDNA contamination; no contamination was detected. The sequences of primers used for qPCR (Kallenbach et alı, 2010; Fragoso et al., 2011) are provided in Table 4.

\section{Quantification of GLV pools in tissue}

To assess qualitatively the GLV pools in leaf tissue from field-grown plants, and to determine appropriate amounts of leaf tissue and internal standard (IS) for GLV extraction, we extracted pooled samples from leaves collected June 6, 2011, from M. sexta-infested plants during infestation M3 (Figure 4). Each sample was pooled from all leaves collected from one genotype. Hexane $(300 \mu \mathrm{L})$ was added to $100 \mathrm{mg}$ tissue spiked with $3 \mu \mathrm{g}$ tetralin as an internal standard (IS) and incubated by rotating at RT overnight. Samples were allowed to settle and $100 \mu \mathrm{L}$ of water- and tissue-free hexane was transferred to a GC vial containing a $250 \mu \mathrm{L}$ microinsert. Individual analytes were analyzed by a Varian CP-3800 GC-Saturn 4000 ion trap MS connected to a ZB5 column $(30 \mathrm{~m} \times 0.25 \mathrm{~mm}$ i.d., $0.25 \mu \mathrm{m}$ film thickness; Phenomenex). $1 \mu \mathrm{L}$ of samples was injected by a CP-8400 autoinjector (Varian) onto the column with a 1:10 split ratio; the injector was returned to a 1:70 split ratio from 2 min after injection through the 


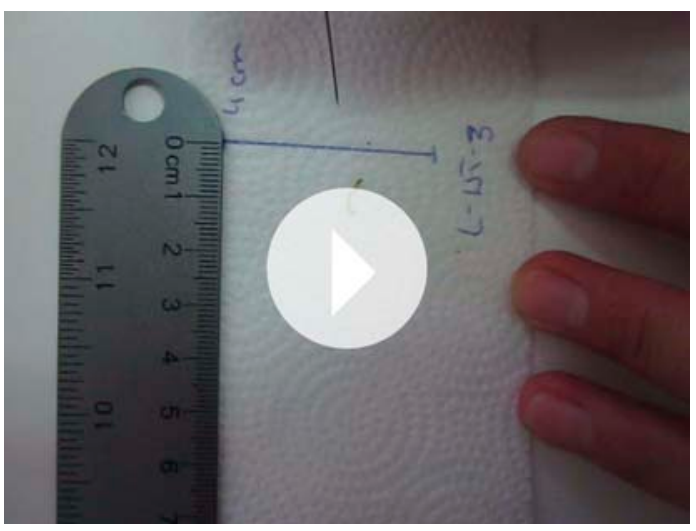

Video 3. Off-plant assay, replicate 3, WT, June 24, 2011. DOI:10.7554/eLife.00007.018 mass spectra databases, Wiley version 6 (Wiley) and NIST (National Institute of Standards and Technology) spectral libraries.

For the quantification of GLV pools in leaf tissue from field-grown plants, the hexane extraction protocol was adjusted based on GC-MS results from pooled samples (described above), and a GC-FID with a wax column was used for the quantitative analysis of extracts. Hexane $(300 \mu \mathrm{L})$ were added to $50 \mathrm{mg}$ tissue ( $\mathrm{N}=10)$ spiked with $15 \mu \mathrm{g}(\mathrm{Z})$-hex-3-enyl acetate, a GLV not found in GC-MS samples, as an IS. The extraction proceeded as described above. Analytes were separated by Varian CP-3800 GC-FID connected to a ZB-Wax column ( $30 \mathrm{~m} \times 0.25 \mathrm{~mm}$ i.d., $0.25 \mu \mathrm{m}$ film thickness; Phenomenex). $1 \mu \mathrm{L}$ of samples was injected by a CP-8400 autoinjector (Varian) onto the column in a splitless mode; the injector was returned to a 1:70 split ratio $2 \mathrm{~min}$ after injection through the end of each run. The GC was programmed as follows: injector held at $230^{\circ} \mathrm{C}$, initial column temperature at $40^{\circ} \mathrm{C}$ held for $7 \mathrm{~min}$, then ramped at $5^{\circ} \mathrm{C} / \mathrm{min}$ to $115^{\circ} \mathrm{C}$ and finally at $30^{\circ} \mathrm{C} / \mathrm{min}$ to $250^{\circ} \mathrm{C}$, held for $0.5 \mathrm{~min}$. Helium carrier gas was used and the column flow set to $1 \mathrm{~mL} / \mathrm{min}$. Compounds eluted from the GC column were transferred to a Varian FID set at $250^{\circ} \mathrm{C}$ for analysis (airflow $300 \mathrm{~mL} / \mathrm{min}$, hydrogen $30 \mathrm{~mL} / \mathrm{min}$, nitrogen make-up gas $5 \mathrm{~mL} / \mathrm{min}$ ). Individual volatile compound peaks were quantified by peak areas using MS Work Station Method Builder and Batch Report software (Varian) and normalized to the peak area of the IS (Z)-hex-3-enyl acetate in each sample. Peak identification and quantification was done by comparison to standard curves of pure compounds in hexane. Compounds present in quantifiable amounts were (Z)-hex-3-en-1-ol, (E)-hex-2-enal and the IS (Z)-hex-3-enyl acetate.

\section{Relative quantification of GLVs and (E)-a-bergamotene in the plant headspace}

For measurement of GLVs in the headspace of field-grown plants, intact leaves were harvested $(N=3)$ and kept fresh by placing the petioles in microcentrifuge tubes filled with water. Immediately before each measurement, one leaf was treated with $\mathrm{W}+\mathrm{OS}$, and a $1 \mathrm{~cm}^{2}$ disc was stamped out and placed in a $4 \mathrm{~mL} \mathrm{GC}$ vial. After $15 \mathrm{~min}$, the headspace in the vial was measured with a ZNose 4200 portable gas chromatograph with a $1 \mathrm{~m}$ DB5 column (Electronic Sensor Technology, Newbury Park, CA, USA) by inserting the ZNose inlet needle through the septum of the $\mathrm{GC}$ vial into the headspace. The program was as follows: valve set at $165^{\circ} \mathrm{C}$, inlet at $200^{\circ} \mathrm{C}$, trap at $250^{\circ} \mathrm{C} ; 30 \mathrm{~s}$ sampling time, column ramped from $30^{\circ} \mathrm{C}$ to $190^{\circ} \mathrm{C}$ at $4^{\circ} \mathrm{C} / \mathrm{s}$, data collection for $20 \mathrm{~s}$. Genotypes were analyzed in an alternating order within each replicate: first replicate 1 of all genotypes, then replicate 2, then replicate 3 . Retention times of GLV aldehydes and alcohols, the most abundant GLV headspace components, were determined using pure standards.

For the analysis of GLVs in the headspace of glasshouse-grown plants, the +2 leaf was enclosed immediately after $\mathrm{W}+\mathrm{OS}$ elicitation in a food-quality $50 \mathrm{~mL}$ plastic container (Huhtamaki) with an activated charcoal filter attached to one side for incoming air, and connected to self-packed Poropak $Q$ filters containing $20 \mathrm{mg}$ of Poropak (Sigma-Aldrich) packed with silanized glass wool and Teflon tubing in the column bodies (ARS, Inc.) as previously described (Halitschke et al., 2000; Schuman et al., 2009). Ambient air was pulled by vacuum pump for $3 \mathrm{hr}$ through an activated charcoal filter, over the leaf in the trapping container, and through a Poropak $Q$ filter connected by PVC tubing (Rotabilo) to a 


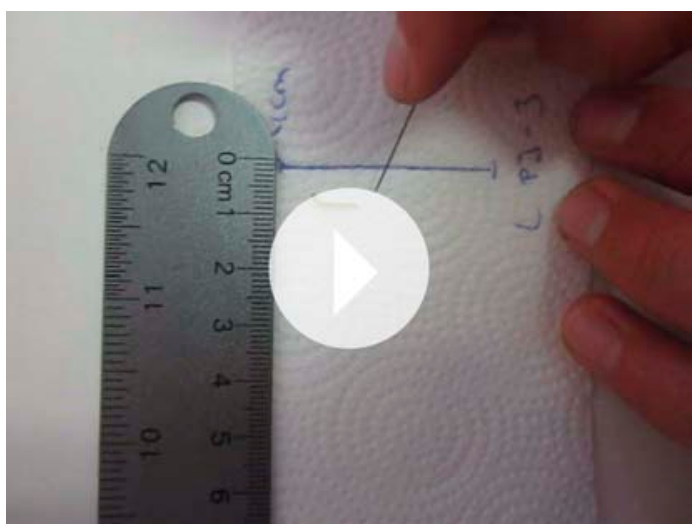

Video 4. Off-plant assay, replicate 3, irPI, June 24, 2011. DOI:10.7554/eLife.00007.019 custom-made valve manifold, as previously described (Schuman et al., 2009); the manifold was adjusted such that flow rates through traps were ca. $300 \mathrm{~mL} / \mathrm{min}$. After trapping, sampled leaves were excised at the base of the petiole, scanned, and the leaf area was measured in comparison to a $1 \mathrm{~cm}^{2}$ standard (SigmaScan 5.0; Systat Software Inc.) for normalization of volatile emission to $\mathrm{cm}^{2}$ leaf area. Poropak $\mathrm{Q}$ filters were wrapped in aluminum foil and stored at $-20^{\circ} \mathrm{C}$ until elution of volatiles with $250 \mu \mathrm{L}$ dichloromethane (Sigma-Aldrich).

Immediately prior to elution, each filter was spiked with $320 \mathrm{ng}$ of tetralin internal standard (IS) in hexane (Sigma-Aldrich). Filters were eluted into a GC vial containing a $250 \mu \mathrm{L}$ glass insert. Samples were analyzed by a CP-3800 GC Varian Saturn 2000 ion trap MS (Varian) connected to a polar ZB-wax column (30 m×0.25 mm i.d., $0.25 \mu \mathrm{m}$ film thickness; Phenomenex). $1 \mu \mathrm{L}$ of samples was injected by a CP-8200 autoinjector (Varian) onto the column in a splitless mode; the injector was returned to a 1:70 split ratio from 2 min after injection through the end of each run. The GC was programmed as follows: injector held at $230^{\circ} \mathrm{C}$, initial column temperature at $40^{\circ} \mathrm{C}$ held for $3 \mathrm{~min}$, then ramped at $5^{\circ} \mathrm{C} / \mathrm{min}$ to $180^{\circ} \mathrm{C}$ and finally at $10^{\circ} \mathrm{C} / \mathrm{min}$ to $240^{\circ} \mathrm{C}$, held for $1 \mathrm{~min}$. Helium carrier gas was used and the column flow set to $1 \mathrm{~mL} / \mathrm{min}$. Eluted compounds from the GC column were transferred to the MS for analysis. The MS was programmed as follows: transfer line at $230^{\circ} \mathrm{C}$, trap temperature $150^{\circ} \mathrm{C}$, manifold temperature $80^{\circ} \mathrm{C}$ and scan range from 40 to $399 \mathrm{~m} / \mathrm{z}$ at 1.33 spectra per second as previously described (Schuman et al., 2009). Individual volatile compound peaks were quantified by peak areas of two specific and abundant ion traces per compound using MS Work Station Data Analysis software (Varian) and normalized by the $104+132$ ion trace peak area of the IS (tetralin) in each sample. The identification of compounds was conducted by GC retention time compared to pure standards and mass spectra compared to standards and mass spectra databases, Wiley version 6 (Wiley) and NIST (National Institute of Standards and Technology) spectral libraries. In $3 \mathrm{hr}$ headspace samples we detected (Z)-hex-3-en-1-ol, (Z)-hex-3-en-1-ol, (E)-hex-2en-1-ol (forms from (E)-hex-2-enal on filters over trapping periods longer than $20 \mathrm{~min}$ ), (Z)-hex-3-enyl acetate, (Z)-hex-3-enyl butanoate, (Z)-hex-3-enyl isobutyrate, and (Z)-hex-3-enyl propanoate.

The collection of (E)-a-bergamotene from the headspace of glasshouse-grown plants and its extraction from Poropak $Q$ filters was carried out as for GLVs, except that (E)-a-bergamotene was collected 24-32 hr after W+OS treatment of the leaf. Eluted samples were analyzed by an HP 6890 GC-5973 quadropole MS (Hewlett-Packard) connected to a nonpolar DB-5ms column (30 m×0.25 mm i.d., $0.25 \mu \mathrm{m}$ film thickness; Agilent). $1 \mu \mathrm{L}$ of samples was injected by a HP 7683 autoinjector (Hewlett-Packard) onto column in a splitless mode; the injector was purged at $50 \mathrm{~mL} / \mathrm{min} 1.5 \mathrm{~min}$ after injection and switched to gas saver mode $(20 \mathrm{~mL} / \mathrm{min})$ from $10 \mathrm{~min}$ through the end of each run. The GC was programmed as follows: injector held at $230^{\circ} \mathrm{C}$, initial column temperature at $40^{\circ} \mathrm{C}$ held for $2 \mathrm{~min}$, then ramped at $5^{\circ} \mathrm{C} / \mathrm{min}$ to $165^{\circ} \mathrm{C}$ and finally at $60^{\circ} \mathrm{C} / \mathrm{min}$ to $300^{\circ} \mathrm{C}$, held for $2 \mathrm{~min}$. Helium carrier gas was used and the column flow set to $2 \mathrm{~mL} / \mathrm{min}$. Eluted compounds from GC column were transferred to the MS for analysis. The MS was programmed as follows: source at $230^{\circ} \mathrm{C}$, quad temperature $150^{\circ} \mathrm{C}$, and scan range from 33 to $350 \mathrm{~m} / \mathrm{z}$ at 4.49 spectra per second. (E)- $\alpha$-Bergamotene was quantified by peak area using the ion trace $119 \mathrm{~m} / \mathrm{z}$ in Chemstation software (Agilent) and normalized by the 104 ion trace peak area of the IS (tetralin) in each sample. The identification of (E)- $\alpha$-Bergamotene and tetralin IS was conducted by GC retention time and mass spectra compared to mass spectra of known standards as previously described (Schuman et al., 2009).

\section{Manduca spp. bioassays}

One or two larvae were placed on plants at a time for each assay, depending on the number available, and were equally distributed among plants as described under 'Manduca spp. infestation and W+OS treatment of plants'. However, for infestation M3 (Figure 4), we staggered the infestation of different plant genotypes to accommodate differences in plant growth: WT and irPI plants were initially larger and therefore went into the field on average earlier than irLOX2 and hemi-irLOX2 plants, so that all 
Table 4. Primers used for quantitative PCR (SYBR Green)

\begin{tabular}{llll} 
Gene & Forward primer sequence $\left(\mathbf{5}^{\prime} \mathbf{-} \mathbf{3}^{\prime}\right)$ & Reverse primer sequence $\left(\mathbf{5}^{\prime} \mathbf{-} \mathbf{3}^{\prime}\right)$ & Citation \\
\hline PI & TCAGGAGATAGTAATATGG & ATCTGCATGTTCCACATTGC & Fragoso et al. (2011) \\
\hline LOX2 & TTGCACTTGGTGTTTGAGATGGT & TTAGTAGAAAATGAGCACCACAA & Kallenbach et al. (2010)
\end{tabular}

DOI: $10.7554 /$ eLife.00007.020

plants were planted at a similar size, which is important for even establishment. We therefore reinfested WT and irPI plants earlier after M1, to allow irLOX2 and hemi-irLOX2 plants to catch up in their growth to WT and irPI before re-infestation. However, we then left M. sexta larvae on irLOX2 and hemi-irLOX2 as long as on WT and irPl, and we used a combination of Geocoris spp. counts and additional predation assays to make sure that differences in Geocoris spp. predation were not due to this staggering of infestation (see 'Results: Geocoris spp. preferentially predate from GLV-perfumed or -emitting plants').

Manduca spp. behavior, predation, and growth assays were conducted with first- and second-instar larvae, except infestations M1 2010 and M4 in 2011, in which larvae were reared from the first through fifth instars on plants; and egg predation assays, in which $M$. sexta eggs were used.

Larvae used in the off-plant mock predation assays were hatched on the appropriate $N$. attenuata genotype (WT or irPl) and hatching was monitored three times per day (morning, noon, evening) so that the mock predation assay could be timed to $48 \mathrm{hr}$ after larvae hatched. A protocol of the mock predation assays is given in Figure 10, and Videos 1-4 depict on-plant (1 and 2) and off-plant (3 and 4) behavioral assays. Larvae for off-plant mock predation assays were kept in aerated plastic boxes on cut leaves over moist paper towels. Leaves were refreshed twice daily and were kept fresh by placing the petioles in water in $1.5 \mathrm{~mL}$ microcentrifuge tubes which were closed around the petiole with Parafilm (Pechiney Plastic Packaging Company). Larval growth was measured as increases in body length (in millimeters) using calipers or a small, flexible, transparent plastic ruler.

\section{Predation assays}

Predation rates were recorded for larvae placed on plants as described above, or for two eggs per plant fixed with droplets of a-cellulose glue (Kessler and Baldwin, 2001) to the underside of a rosette or lower stem leaf at a standardized position. For egg predation assays, a wild Manduca spp. larva was enclosed on a nearby leaf to ensure continual GLV emission: a clip cage was closed around the larva to make it inaccessible to Geocoris spp. predators. Predation was monitored mornings and evenings. Larvae were considered to be predated when either the larva was missing over multiple days, but clear Manduca spp. feeding damage was present, or when the predated larval carcass was found (Figure 5). Mortality was defined as the total number of missing larvae. Eggs were considered predated when the eggshell was empty but intact except for a small hole which characterizes the typical damage caused by Geocoris spp. feeding; eggs occasionally collapse during Geocoris spp. predation, but collapsed eggs were not counted unless the eggs were mostly or fully empty and with a visible hole (Figure 5).

\section{GLV supplementation}

During infestation M2, GLVs were added back to irLOX2 and hemi-irLOX2 headspaces by placing a cotton swab adjacent to the $M$. sexta-infested leaf and adding ca. $20 \mu \mathrm{L}$ of lanolin paste, measured with a seed spoon, containing a mix of pure GLVs representative of the $M$. sexta-fed headspace and dissolved in hexane (Table 1) (Allmann and Baldwin, 2010) to the cotton swab. Cotton swabs bearing ca. $20 \mu \mathrm{L}$ of lanolin paste with hexane as a control were placed next to $M$. sexta-infested leaves of WT and irPI plants. Lanolin pastes were regularly refreshed by adding $20 \mu \mathrm{L}$ in the early afternoon and in the morning. Placing GLVs next to, rather than on the leaf ensured that the supplemented headspace would not be altered by plant metabolism, and that we could terminate the supplementation by removing the cotton swabs.

\section{Geocoris spp. counts}

Field plots were monitored daily for Geocoris spp. presence during the experiments in 2010 and 2011; both G. pallens and G. punctipes were present in 2011, but most individuals observed on and around plants were G. pallens. Soon after the first Geocoris spp. sightings in May 2011 (before 
the first infestation, M2), Geocoris spp. populations in the immediate vicinity of experimental plants were monitored every 2-3 days by counting individuals. Counts were conducted during the main period of Geocoris spp. activity in the early afternoon, by at least two observers in parallel, in order to complete the count around all Manduca spp.-infested and control plants within 20-30 min. Each observer proceeded by looking at a focal plant and its immediate vicinity for $15 \mathrm{~s}$ and then quickly inspecting the rosette leaves; all Geocoris spp. adults and nymphs seen on, under, or within $5 \mathrm{~cm}$ around the rosette of the plant during this time were counted. Observers moved in synchrony with each other from one end of the field plot to the other, in this way counting predators around plants which had not yet been disturbed.

\section{Plant growth and reproduction}

Plant size (rosette diameter, stem length and branching) was monitored at the end of infestation M1 in 2010 and from the beginning of infestation M2 in 2011 (Figure 4): rosette diameter was measured as the maximum diameter found by gently laying a ruler over the rosette; stem length was measured from the base of the stem to the tip of the apical inflorescence by placing a ruler beside the stem; and all side branches $5 \mathrm{~cm}$ or longer were counted. Reproductive output was monitored by counting the number of closed flowers removed every 2-3 days (before they opened) from the beginning of flowering, and by counting numbers of closed flowers and buds $2 \mathrm{~mm}$ or larger at the end of infestation M1 in 2010, and during all infestations in 2011. All growth and reproduction data were analyzed for differences in control versus M. sexta-infested plants within each genotype (statistics Table 3). Because size-matched plants had been planted over one week in 2011, growth and reproduction data from plants in 2011 were organized by the number of days since planting for comparison between genotypes (Figure 6).

\section{Removal of flowers during infestations $M 1$ and M3, and of reproductive meristems prior to infestation M4}

Flowers were removed and counted periodically over the first 10 days of infestation M1 and during infestation M3 (Figures 4 and 6, statistics Table 3), in order to track plant reproduction while avoiding ripe seed capsules: the distribution of ripe seed is not permitted for genetically modified plants. For infestation M4 in 2011 (Figure 4), instead of regularly removing flowers, we removed all reproductive meristems by cutting inflorescences, which contained mostly buds, at their base, so that we would be able to follow a new set of reproductive meristems through to seed set without incurring ripe seed. Because plants were matched prior to M4 and had the same number of buds and of side branches (which usually terminate in inflorescences) (Figure 8, see 'Discussion'), all plants were similarly affected by the removal of reproductive meristems.

\section{Herbivore damage and plant health}

Photographs were taken of entire plants and $M$. sexta-damaged leaves during infestations M2 and M3 in 2011. Damage caused by M. sexta larvae was rated from photographs by an independent observer with no knowledge of plant identity. Total percent canopy damage due to $M$. sexta was rated as 1,2 , 3, or 4 using the damage index in Figure 7.

We monitored herbivore attack to determine whether GLV-silenced plants suffered different amounts of herbivore damage, which could influence fitness measurements. The naturally occurring herbivore community on plants in 2010 and 2011 comprised mirids (Tupiocoris notatus) and noctuid larvae; in 2011 grasshoppers (Trimerotropis spp.) and flea beetles (Epitrix spp.) were also present. Total canopy damage due to herbivores occurring naturally on the field plot was quantified prior to the infestations in 2010 and 2011 and again during infestation M3 in 2011. Damage was calculated by identifying damage from specific herbivores according to their characteristic feeding patterns, counting the number of leaves per plant (small leaves were counted as $1 / 5$ to $1 / 2$ of a leaf based on leaf area and large leaves were counted as 1 leaf), estimating the total percentage of leaf area damage due to each herbivore, and dividing the total leaf area damage from each herbivore by the total number of leaves. Leaf area damage was estimated in categories of $1 \%, 5 \%, 10 \%, 15 \%$, and so on, in steps of $5 \%$. All such damage estimates were made by MCS or KB, who first practiced quantifying damage together until they consistently arrived at the same numbers.

As part of matching plants prior to infestation M4 in 2011, plant health was rated on a scale of 1 (dead) to 5 (healthy) using the index in Figure 7. 


\section{Statistical analyses}

Fisher's exact tests were conducted using a macro (J H Macdonald, http://udel.edu/ mcdonald/ statfishers.html) for Excel (Microsoft). All other statistical analyses were conducted with SPSS 17.0 (IBM). Count data were analyzed either by Fisher's exact tests (independent values) or by Friedman tests (repeated measures). Levene's test for homogeneity of variance was performed prior to all t-tests and ANOVAs and when necessary, data were $\log _{2}$ transformed (volatile and transcript data), square root transformed (count data) or arcsin transformed (herbivore damage data) to meet requirements for homogeneity of variance. Parametric data were compared using ANOVAs, MANOVAs, or repeatedmeasure ANOVAs followed by Scheffe post hoc tests. If variance was not homogeneous following transformation, data were compared using Kruskal-Wallis tests (for multiple comparisons) or Mann-Whitney U-tests (for two-way comparisons) and Bonferroni p-value corrections were used to correct for nonparametric multiple comparisons. For Kruskal-Wallis tests and Mann-Whitney U-tests, a Monte Carlo algorithm was used with 10,000 permutations and a 95\% confidence level.

\section{Acknowledgements}

We thank S Allmann for conducting egg predation assays in 2010; C Diezel, M Erb, M Kallenbach, D Kessler, D Marciniak, M Stanton, and A Steppke for help with field work; S Weigl for rating M. sexta damage levels; J Gershenzon for the use of the HP GC-MS; M Erb, J Gershenzon, D Heckel, M Kallenbach, A Kessler, A Steppke, and A Weinhold for comments on the manuscript; Brigham Young University for the use of their Lytle Preserve field station; and APHIS for constructive regulatory oversight.

\section{Additional information}

Competing interests

ITB: Senior Editor, eLife. The other authors have declared that no competing interests exist.

Funding

\begin{tabular}{ll} 
Funder & Author \\
\hline Max Planck Society & Meredith C Schuman, \\
& Kathleen Barthel, \\
& lan T Baldwin \\
\hline
\end{tabular}

The funder had no role in study design, data collection and interpretation, or the decision to submit the work for publication.

\section{Author contributions}

MCS, Conception and design, acquisition of data, analysis and interpretation of data, and drafting or revising the article; KB, Conception and design, acquisition of data, analysis and interpretation of data, and drafting or revising the article; ITB, Conception and design, acquisition of data, analysis and interpretation of data, and drafting or revising the article.

\section{Additional files}

Major datasets

The following datasets were generated

\begin{tabular}{lllll}
\hline Author(s) & Year & Dataset title & Dataset ID and/or URL & $\begin{array}{l}\text { Database, license, and } \\
\text { accessibility information }\end{array}$ \\
\hline Schuman MC, Barthel K, Baldwin IT & 2012 & $\begin{array}{l}\text { Herbivory-induced volatiles } \\
\text { function as defenses } \\
\text { increasing fitness of the } \\
\text { native plant Nicotiana } \\
\text { attenuata in nature }\end{array}$ & $\begin{array}{l}\text { http://dx.doi.org/10.5061/ } \\
\text { dryad.gs45f }\end{array}$ & $\begin{array}{l}\text { Available at Dryad Digital } \\
\text { Repository under a CC0 } \\
\text { Public Domain Dedication }\end{array}$ \\
& & &
\end{tabular}




\section{References}

Allison JD, Hare JD. 2009. Learned and naïve natural enemy responses and the interpretation of volatile organic compounds as cues or signals. New Phytol 184: 768-782. doi:10.1111/j.1469-8137.2009.03046.x.

Allmann S, Baldwin IT. 2010. Insects betray themselves in nature to predators by rapid isomerization of green leaf volatiles. Science 329: 1075-1078. doi:10.1126/science.1191634.

Allmann S, Halitschke R, Schuurink RC, Baldwin IT. 2010. Oxylipin channelling in Nicotiana attenuata: Lipoxygenase 2 supplies substrates for green leaf volatile production. Plant Cell Environ 33: 2028-2040. doi:10.1111/j.1365-3040.2010.02203.x.

Baldwin IT. 1998. Jasmonate-induced responses are costly but benefit plants under attack in native populations. Proc Natl Acad Sci U S A, 95: 8113-8118.

Benrey B, Denno RF. 1997. The slow-growth-high mortality hypothesis: A test using the cabbage butterfly. Ecology 78: 987-999. doi:10.2307/2265852.

Bubner B, Gase K, Berger B, Link D, Baldwin IT. 2006. Occurrence of tetraploidy in Nicotiana attenuata plants after Agrobacterium-mediated transformation is genotype specific but independent of polysomaty of explant tissue. Plant Cell Rep 25: 668-675. doi:10.1007/s00299-005-0111-4.

De Moraes CM, Mescher MC, Tumlinson JH. 2001. Caterpillar-induced nocturnal plant volatiles repel conspecific females. Nature 410: 577-580. doi:10.1038/35069058.

Degenhardt J, Hiltpold I, Köllner TG, Frey M, Gierl A, Gershenzon J, et al. 2009. Restoring a maize root signal that attracts insect-killing nematodes to control a major pest. Proc Natl Acad Sci U S A 106: 13213-13218. doi:10.1073/pnas.0906365106.

Dicke M, Baldwin IT. 2010. The evolutionary context for herbivore-induced plant volatiles: Beyond the 'cry for help'. Trends Plant Sci 15: 167-175. doi:10.1016/j.tplants.2009.12.002.

Eubanks MD, Denno RF. 1999. The ecological consequences of variation in plants and prey for an omnivorous insect. Ecology 80: 1253-1266. doi:10.2307/177072.

Eubanks MD, Denno RF. 2000. Host plants mediate omnivore-herbivore interactions and influence prey suppression. Ecology 81: 936-947. doi:10.2307/177168.

Fragoso V, Goddard H, Baldwin IT, Kim S-G. 2011. A simple and efficient micrografting method for stably transformed Nicotiana attenuata plants to examine shoot-root signaling. Plant Methods 7: 34. doi:10.1186/1746-4811-7-34.

Fraser AM, Mechaber WL, Hildebrand JG. 2003. Electroantennographic and behavioral responses of the sphinx moth Manduca sexta to host plant headspace volatiles. J Chem Ecol 29: 1813-1833. doi: 10.1023/A:1024898127549.

García-Pérez RD, Houdt HV, Depicker A. 2004. Spreading of post-transcriptional gene silencing along the target gene promotes systemic silencing. Plant J 38: 594-602. doi:10.1111/j.1365-313X.2004.02067.x.

Halitschke R, Baldwin IT. 2003. Antisense LOX expression increases herbivore performance by decreasing defense responses and inhibiting growth-related transcriptional reorganization in Nicotiana attenuata. Plant $J$ 36: 794-807. doi:10.1046/j.1365-313X.2003.01921.x.

Halitschke R, Kessler A., Kahl J, Lorenz A, Baldwin IT. 2000. Ecophysiological comparison of direct and indirect defenses in Nicotiana attenuata. Oecologia 124: 408-417. doi:10.1007/s004420000389.

Halitschke R, Schittko U, Pohnert G, Boland W, Baldwin IT. 2001. Molecular interactions between the specialist herbivore Manduca sexta (Lepidoptera, Sphingidae) and its natural host Nicotiana attenuata. III. Fatty acidamino acid conjugates in herbivore oral secretions are necessary and sufficient for herbivore-specific plant responses. Plant Physiol 125: 711-717. doi: 10.1104/pp.125.2.711.

Halitschke R, Ziegler J, Keinänen M, Baldwin IT. 2004. Silencing of hydroperoxide lyase and allene oxide synthase reveals substrate and defense signaling crosstalk in Nicotiana attenuata. Plant J 40: 35-46. doi:10.1111/j.1365-313X.2004.02185.x.

Halitschke R, Stenberg JA, Kessler D, Kessler A, Baldwin IT. 2008. Shared signals_'alarm calls' from plants increase apparency to herbivores and their enemies in nature. Ecol Lett 11: 24-34. doi:10.1111/j.1461-0248. 2007.01123.x.

Hartl M, Giri AP, Kaur H, Baldwin IT. 2011. The multiple functions of plant serine protease inhibitors: Defense against herbivores and beyond. Plant Signal Behav 6: 1009-1011. doi:10.4161/psb.6.7.15504.

Heil M. 2008. Indirect defence via tritrophic interactions. New Phytol 178: 41-61. doi:10.1111/j.1469-8137.2007.02330.x.

Hoballah MEF, Turlings TCJ. 2001. Experimental evidence that plants under caterpillar attack may benefit from attracting parasitoids. Evol Ecol Res 3: 553-565.

Horne P., Page J. 2008. Integrated pest management for crops and pastures. Landlinks Press, Victoria.

Kallenbach M, Alagna F, Baldwin IT, Bonaventure G. 2010. Nicotiana attenuata SIPK, WIPK, NPR1, and fatty acid-amino acid conjugates participate in the induction of jasmonic acid biosynthesis by affecting early enzymatic steps in the pathway. Plant Physiol 152: 96-106. doi:10.1104/pp.109.149013.

Kaplan I. 2012. Attracting carnivorous arthropods with plant volatiles: The future of biocontrol or playing with fire? Biol Control 60: 77-89. doi:10.1016/j.biocontrol.2011.10.017.

Kaplan I, Thaler JS. 2011. Do plant defenses enhance or diminish prey suppression by omnivorous Heteroptera? Biol Control 59: 53-60. doi:10.1016/j.biocontrol.2010.12.005.

Kappers IF, Aharoni A, van Herpen TWJM, Luckerhoff LLP, Dicke M, Bouwmeester HJ. 2005. Genetic engineering of terpenoid metabolism attracts bodyguards to Arabidopsis. Science 309: 2070-2072. doi:10.1126/ science.1116232. 
Karban R, Baldwin IT. 1997. Induced responses to herbivory. University of Chicago Press, Chicago.

Kessler A, Baldwin IT. 2001. Defensive function of herbivore-induced plant volatile emissions in nature. Science 291: 2141-2144. doi:10.1126/science.291.5511.2141.

Kessler A, Baldwin IT. 2004. Herbivore-induced plant vaccination. Part I. The orchestration of plant defenses in nature and their fitness consequences in the wild tobacco Nicotiana attenuata. Plant J 38: 639-649. doi:10.1111/j.1365-313X.2004.02076.x.

Kessler D, Baldwin IT. 2006. Making sense of nectar scents: The effects of nectar secondary metabolites on floral visitors of Nicotiana attenuata. Plant J 49: 840-854. doi:10.1111/j.1365-313X.2006.02995.x.

Kessler A, Heil M. 2011. The multiple faces of indirect defences and their agents of natural selection. Funct Ecol 25: 348-357. doi:10.1111/j.1365-2435.2010.01818.x.

Kessler A, Halitschke R, Baldwin IT. 2004. Silencing the jasmonate cascade: Induced plant defenses and insect populations. Science 305: 665-668. doi:10.1126/science.291.5511.2141.

Kessler A, Halitschke R, Diezel C, Baldwin IT. 2006. Priming of plant defense responses in nature by airborne signaling between Artemisia tridentata and Nicotiana attenuata. Oecologia 148: 280-292. doi:10.1007/ s00442-006-0365-8.

Kos M, van Loon JJA, Dicke M, Vet LEM. 2009. Transgenic plants as vital components of integrated pest management. Trends Biotechnol 27: 621-627. doi:10.1016/j.tibtech.2009.08.002.

Krügel T, Lim M, Gase K, Halitschke R, Baldwin IT. 2002. Agrobacterium-mediated transformation of Nicotiana attenuata, a model ecological expression system. Chemoecology 12: 177-183. doi:10.1007/ PL00012666.

Liu F, Xu Z, Zhu YC, Huang F, Wang Y, Li H, et al. 2010. Evidence of field-evolved resistance to Cry1Ac-expressing Bt cotton in Helicoverpa armigera (Lepidoptera: Noctuidae) in northern China. Pest Manag Sci 66: 155-161. doi:10.1002/ps.1849.

Meldau S, Wu J, Baldwin IT. 2009. Silencing two herbivory-activated MAP kinases, SIPK and WIPK, does not increase Nicotiana attenuata's susceptibility to herbivores in the glasshouse and in nature. New Phytol 181: 161-173. doi:10.1111/j.1469-8137.2008.02645.x.

Paschold A, Halitschke R, Baldwin IT. 2006. Using 'mute' plants to translate volatile signals. Plant J 45: 275-291. doi:10.1111/j.1365-313X.2005.02623.x.

Price PW, Bouton CE, Gross P, McPheron BA, Thompson JN, Weis AE. 1980. Interactions among three trophic levels: Influence of plants on interactions between insect herbivores and natural enemies. Ann Rev Ecol Syst 11: 41-65. doi:10.1146/annurev.es.11.110180.000353.

Rasmann S, Köllner TG, Degenhardt J, Hiltpold I, Toepfer S, Kuhlmann U, et al. 2005. Recruitment of entomopathogenic nematodes by insect-damaged maize roots. Nature 434: 732-737. doi:10.1038/nature03451.

Schittko U, Preston CA, Baldwin IT. 2000. Eating the evidence? Manduca sexta larvae can not disrupt specific jasmonate induction in Nicotiana attenuata by rapid consumption. Planta 210: 343-346. doi:10.1007/ PL00008143.

Schittko U, Hermsmeier D, Baldwin IT. 2001. Molecular interactions between the specialist herbivore Manduca sexta (Lepidoptera, Sphingidae) and its natural host Nicotiana attenuata. II. Accumulation of plant mRNAs in response to insect-derived cues. Plant Physiol 125: 701-710. doi:10.1104/pp.125.2.701.

Schnee C, Köllner TG, Held M, Turlings TCJ, Gershenzon J, Degenhardt J. 2006. The products of a single maize sesquiterpene synthase form a volatile defense signal that attracts natural enemies of maize herbivores. Proc Natl Acad Sci U S A 103: 1129-1134. doi:10.1073/pnas.0508027103.

Schuman M, Heinzel N, Gaquerel E, Svatos A, Baldwin IT. 2009. Polymorphism in jasmonate signaling partially accounts for the variety of volatiles produced by Nicotiana attenuata plants in a native population. New Phytol 183: 1134-1148. doi:10.1111/j.1469-8137.2009.02894.x.

Schuman M, Barthel K, Baldwin IT. 2012. Data from: Herbivory-induced volatiles function as defenses increasing fitness of the native plant Nicotiana attenuata in nature. Dryad Digital Repository. http://dx.doi.org/10.5061/ dryad.gs $45 f$.

Schwachtje J, Kutschbach S, Baldwin IT. 2008. Reverse genetics in ecological research. PLoS One 3: e1543. doi:10.1371/journal.pone.0001543.

Sime KR, Baldwin IT. 2003. Opportunistic out-crossing in Nicotiana attenuata (Solanaceae), a predominantly self-fertilizing native tobacco. BMC Ecol 3: 6. doi:10.1186/1472-6785-3-6.

Skibbe M, Qu N, Galis I, Baldwin IT. 2008. Induced plant defenses in the natural environment: Nicotiana attenuata WRKY3 and WRKY6 coordinate responses to herbivory. Plant Cell 20: 1984-2000. doi:10.1105/ tpc.108.058594.

Steppuhn A, Baldwin IT. 2007. Resistance management in a native plant: Nicotine prevents herbivores from compensating for plant protease inhibitors. Ecol Lett 10: 499-511. doi:10.1111/j.1461-0248.2007.01045.x.

Stork WFJ, Weinhold A, Baldwin IT. 2011. Trichomes as dangerous lollipops: Do lizards also use caterpillar body and frass odor to optimize their foraging? Plant Signal Behav 6: 1893-1896. doi:10.4161/psb.6.12.18028.

Travella S, Klimm TE., Keller B. 2006. RNA interference-based gene silencing as an efficient tool for functional genomics in hexaploid bread wheat. Plant Physiol 142: 6-20. doi:10.1104/pp.106.084517.

Turlings TCJ, Wäckers FL. 2004. Recruitment of predators and parasitoids by herbivore-injured plants. In Advances in insect chemical ecology (eds Cardé RT, Millar JG), pp. 21-75. Cambridge University Press, Cambridge.

van Dam NM, Horn M, Mares M, Baldwin IT. 2001. Ontogeny constrains systemic protease inhibitor response in Nicotiana attenuata. J Chem Ecol 27: 547-568. doi:10.1023/A:1010341022761. 
van Loon JJA, de Boer JG, Dicke M. 2000. Parasitoid-plant mutualism: Parasitoid attack of herbivore increases plant reproduction. Entomologia Experimentalis et Applicata, 97, 219-227. doi:10.1046/j.1570-7458.2000.00733.x.

Weinhold A, Baldwin IT. 2011. Trichome-derived O-acyl sugars are a first meal for caterpillars that tags them for predation. Proc Natl Acad Sci U S A 108: 7855-7859. doi:10.1073/pnas.1101306108.

Williams IS. 1999. Slow-growth, high-mortality-a general hypothesis, or is it? Ecol Entomol 24: 490-495. doi:10.1046/j.1365-2311.1999.00217.x.

Wink M, Theile V. 2002. Alkaloid tolerance in Manduca sexta and phylogenetically related sphingids (Lepidoptera: Sphingidae). Chemoecology 46: 29-46. doi:10.1007/s00049-002-8324-2.

Winterer J., Bergelson J. 2001. Diamondback moth compensatory consumption of protease inhibitor-transformed plants. Mol Ecol 10: 1069-1074. doi:10.1046/j.1365-294X.2001.01239.x.

Wu J, Hettenhausen C, Baldwin IT. 2006. Evolution of proteinase inhibitor defenses in North American allopolyploid species of Nicotiana. Planta 224: 750-760. doi:10.1007/s00425-006-0256-6.

Zavala JA, Baldwin IT. 2004. Fitness benefits of trypsin proteinase inhibitor expression in Nicotiana attenuata are greater than their costs when plants are attacked. BMC Ecol 4: 11. doi:10.1186/1472-6785-4-11.

Zavala JA, Giri AP, Jongsma MA, Baldwin IT. 2008. Digestive duet: Midgut digestive proteinases of Manduca sexta ingesting Nicotiana attenuata with manipulated trypsin proteinase inhibitor expression. PLoS One 3: 29-31. doi:10.1371/journal.pone.0002008. 